\title{
Exponential stability for delayed complex-valued neural networks with reaction-diffusion terms
}

\author{
Xiaohui $X u^{1,2} \mathbb{D}$, Jibin Yang ${ }^{2 *}$, Quan $X u^{3}$, Yanhai $X u^{2}$ and Shulei Sun ${ }^{2}$
}

\section{"Correspondence:}

yangjibin08@163.com

${ }^{2}$ Key Laboratory of Automobile

Measurement and Control \& Safty,

School of Automobile \&

Transportation, Xihua University,

Chengdu, 610039, China

Full list of author information is

available at the end of the article

\section{Springer}

\begin{abstract}
In this study, we investigate reaction-diffusion complex-valued neural networks with mixed delays. The mixed delays include both time-varying and infinite distributed delays. Criteria are derived to ensure the existence, uniqueness, and exponential stability of the equilibrium state of the addressed system on the basis of the M-matrix properties and homeomorphism mapping theories as well as the vector Lyapunov function method. The results demonstrate the positive effect of reaction-diffusion on the stability, which further improves the existing conditions. Finally, the analysis of several examples is compared to the present results to verify the correctness and reduced conservatism of the primary results.
\end{abstract}

Keywords: Complex-valued neural networks; Reaction-diffusion terms; Time-varying delays; Infinite distributed delays; Exponential stability; Vector Lyapunov function method

\section{Introduction}

Because there are many potential applications of CVNNs [1-4], there has been increasing interest in research and development related to complex-valued neural networks $(\mathrm{CVNNs})$, in which neuron states and connection matrices are defined in the complex number domain. For instance, one typical application of CVNNs is image processing using the two dimensional value of the image signal, which is regarded as a complex number. Considering that applications of CVNNs are based on dynamic behaviors including bifurcations, stability, and chaos, research related to the dynamic behaviors of various CVNNs has become increasingly popular [5-29].

It is well known that time delays must exist in neural networks (NNs) during hardware processing. Therefore, it is important to include these delays in models of NNs. Constant delays were considered in models of CVNNs in [6, 7, 11-14, 23, 27, 28]. Because the time delays continuously change in practical systems, the scholars in $[9,10,15-$ $18,22,24,25,29]$ established various types of CVNNs with time-varying delays and proposed some sufficient conditions for judging the dynamical behaviors of the system states. Furthermore, infinite distributed delays were also introduced into the models of CVNNs in $[5,19-21]$ because NNs generally have a spatial extent because of the presence of a

(c) The Author(s) 2021. This article is licensed under a Creative Commons Attribution 4.0 International License, which permits use sharing, adaptation, distribution and reproduction in any medium or format, as long as you give appropriate credit to the original author(s) and the source, provide a link to the Creative Commons licence, and indicate if changes were made. The images or other third party material in this article are included in the article's Creative Commons licence, unless indicated otherwise in a credit line to the material. If material is not included in the article's Creative Commons licence and your intended use is not permitted by statutory regulation or exceeds the permitted use, you will need to obtain permission directly from the copyright holder. To view a copy of this licence, visit http://creativecommons.org/licenses/by/4.0/. 
multitude of parallel pathways with a variety of axon sizes and lengths, and the way to incorporate this is to introduce infinite distributed delays.

In biological NNs, the existence of diffusion is generally unavoidable due to the inhomogeneity of the cell concentration. The diffusion effect can cause the system to produce some new spatial phenomena including chaos, spiral waves, and Turing instability. Thus, investigating the dynamical behaviors of NNs with reaction-diffusion terms is very important. Therefore, many scholars have conducted significant amounts of research that have produced many significant results [28-44]. In [31, 33, 39], several classes of time-varying delays NNs were put forward, and the corresponding theorems for judging the stability of the equilibrium states of these systems were proposed by applying the Lyapunov function method and LMI technique. Using a similar method as that proposed by [31, 33, 39], stability analysis of the equilibrium state was performed for Cohen-Grossberg NNs with reaction-diffusion terms as well as mixed delays including time-varying and infinite distributed delays $[32,35]$. In contrast, using a methodology different from that adopted in $[31-33,35,39]$ that combined the Lyapunov function method with M-matrix theory, in $[30,40,42]$ the authors presented some important stability results that could be used to judge reaction-diffusion neural networks (RDNNs) with mixed delays as well as Markova jumping parameters [30] or random disturbances [40, 42].

It is noteworthy that the results proposed in [30-42] and the references therein only work for real-valued neural networks (RVNNs). However, not many researchers have studied the dynamical behaviors of CVNNs with reaction-diffusion terms. To our knowledge, there are only two published papers $[28,29]$ that are concerned with the dynamical behavior of reaction-diffusion CVNNs (RDCVNNs). In [28], a class of delayed RDCVNNs under Dirichlet boundary conditions was considered. The dynamical behaviors affecting the stability as well as the Hopf bifurcation of the equilibrium state were studied based on normal form theory and the center manifold theorem of partial differential equations. In [29], the authors investigated two types of coupled memristive CVNNs with diffusion terms, and derived several novel criteria for ensuring passivity of these two networks by exploiting some inequality techniques, a Lyapunov functional approach, and the construction of suitable controller. Fixed and time-varying delays were considered in [28] and [29], respectively. However, mixed delays including time-varying and infinite distributed delays have not been considered in RDCVNN models, and it is necessary to study this further.

Among the methods used to analyze the dynamics of NNs, the one most commonly adopted by scholars is the scalar Lyapunov function method combined with LMI techniques $[6,12,14,16,17,19,21,23-25,31-33,35,39]$. Due to the appearance of free variables within the proposed criteria, the conservatism of the criteria reduces theoretically. Nevertheless, as pointed out in [45], the perfect composition in real applications between the supposition-based parameters of the NNs and incoming free variables depends on experience and incessant attempts to a great degree. Without using LMI techniques, the scalar Lyapunov function method was applied to established some criteria for judging the stability of the corresponding RVNNs in [34, 36, 37, 40,41] and CVNNs in $[8-11,13,18]$. Similar problems concerning the stability conditions obtained by the scalar Lyapunov function method combined with LMI techniques existed because of the relax variables that were introduced. Another method called the vector Lyapunov function method was chosen to study a class of CVNNs in [5]. The stability conditions [5] had 
a compact matrix form that did not include any free variables, and they could be easily calculated to verify the stability of the systems.

A summary of the above references indicates that, to date, there have been no published papers that describe applying the vector Lyapunov function approach to the stability analysis of a type of delayed RDCVNNs with both time-varying and infinite distributed delays. Inspired by this analysis, the purpose of this study is to investigate the existence, uniqueness, and global exponential stability for RDCVNNs with mixed delays. The major contributions of this study are: (1) models of RDCVNNs with mixed delays are considered, which include related existing models; (2) a new lemma is proposed to process the reaction-diffusion terms of $\mathrm{NNs}$, which demonstrates a stronger positive influence of the reaction-diffusion terms on stability than that of the lemmas in [32, 36, 37, 40]; (3) by adopting the vector Lyapunov function method combined with the M-matrix properties and the homeomorphism mapping theory, some sufficient conditions without any relax variables are established for ensuring the existence, uniqueness, and global exponential stability of the equilibrium state of the proposed system; (4) the established stability conditions exhibit compacted matrix forms, which do not depend on an individual attempt; and (5) finally, several numerical examples are provided to demonstrate the feasibility and lower-level conservatism of the established results as compared with the previously existing ones.

This paper includes five sections. Section 2 provides a description of the model and preliminaries, Sect. 3 gives several theorems and corollaries, and Sect. 4 provides several examples. Finally, the conclusions of this study and some directions for future study are proposed in Sect. 5.

\section{Preliminaries}

For convenience, some common notations are used throughout this paper. Let $\mathbb{C}$ denote a complex number set. Let $\tilde{u}=\tilde{u}^{R}+\tilde{u}^{I} i$ be a complex number, where $i$ denotes an imaginary unit, i.e. $i^{2}=-1$. Let $\Theta=\{1,2, \ldots, n\}$ denote a finite set. For a complex number vector $\tilde{\mathbf{u}} \in \mathbb{C}^{n}$, let $|\tilde{\mathbf{u}}|=\left(\left|\tilde{u}_{1}\right|,\left|\tilde{u}_{2}\right|, \ldots,\left|\tilde{u}_{n}\right|\right)^{T}$ be the module of the vector $\tilde{\mathbf{u}}$, where $(\cdot)^{T}$ denotes the transpose of the vector. Assuming that $\Omega \in \mathbb{R}^{m}$ is a bounded compact set with a smooth boundary $\partial \Omega$ and $\operatorname{mes}(\Omega)>0$ in space $\mathbb{R}^{m}$. Let $\tilde{u}_{k}(t, \mathbf{z})$ be the state variable of the $k$ th neuron in space $\mathbf{z}$ and at time $t$, where $\mathbf{z} \in \Omega, t>0, k \in \Theta$. The symbol $L^{2}(\mathbb{C} \times \Omega)$ denotes the complex number space of Lebesgue measurable functions of $\mathbb{C} \times \Omega$, and $\left\|\tilde{u}_{k}(t, \mathbf{z})\right\|_{L^{2}}=$ $\left(\int_{\Omega}\left|\tilde{u}_{k}(t, \mathbf{z})\right|^{2} \mathrm{~d} \mathbf{z}\right)^{1 / 2}<\infty, k \in \Theta$.

Consider a type of RDCVNNs that can be described as follows:

$$
\left\{\begin{aligned}
& \frac{\partial \tilde{u}_{k}(t, \mathbf{z})}{\partial t}= \pi_{k} \sum_{h=1}^{m} \frac{\partial}{\partial z_{h}}\left(\frac{\partial \tilde{u}_{k}(t, \mathbf{z})}{\partial z_{h}}\right)-d_{k} \tilde{u}_{k}(t, \mathbf{z})+\sum_{j=1}^{n}\left[a_{k j} \tilde{g}_{j}\left(\tilde{u}_{j}(t, \mathbf{z})\right)\right. \\
&\left.+b_{k j} \tilde{g}_{j}\left(\tilde{u}_{j}\left(t-\tau_{k j}(t)\right), \mathbf{z}\right)+q_{k j} \int_{-\infty}^{t} \sigma_{k j}(t-s) \tilde{g}_{j}\left(\tilde{u}_{j}(s, \mathbf{z})\right) \mathrm{d} s\right]+J_{k} \\
&\left.\tilde{u}_{k}(t, \mathbf{z})\right|_{\mathbf{z} \in \partial \Omega}=0
\end{aligned}\right.
$$

where $t \geq 0, k \in \Theta, n \geq 2$ denotes the number of neurons. $\tilde{u}_{k}(t, \mathbf{z})$ with $\mathbf{z} \in \Omega$ represents the state variable of $k$ th neuron at time $t . \pi_{k}>0$ denotes the transmission-diffusion coefficient. Let $z_{h}$ represent the space variable, where $h=1,2, \ldots, m . d_{k}>0$ denotes the charging time constant or passive decay rate of the $k$ th neuron. $\mathbf{A}=\left(a_{k j}\right)_{n \times n}, \mathbf{B}=\left(b_{k j}\right)_{n \times n}$, and $\mathbf{Q}=\left(q_{k j}\right)_{n \times n}$ are the connection weight matrices and they are defined in $\mathbb{C}^{n \times n}$. $\tilde{\mathbf{g}}(\tilde{\mathbf{u}})=$ 
$\left(\tilde{g}_{1}\left(\tilde{u}_{1}\right), \tilde{g}_{2}\left(\tilde{u}_{2}\right), \ldots, \tilde{g}_{n}\left(\tilde{u}_{n}\right)\right)^{T}$ represents the activation function of the neurons. $J_{k}$ denotes the constant external input on the $k$ th neuron.

Let $\mathbf{J}=\left(J_{1}, J_{2}, \ldots, J_{n}\right)^{T}, \boldsymbol{\pi}=\operatorname{diag}\left(\pi_{k}\right)_{n \times n}$, and $\mathbf{D}=\operatorname{diag}\left(d_{k}\right)_{n \times n}$.

Let the initial conditions of (1) be $\tilde{u}_{k}(s, \mathbf{z})=\tilde{\varphi}_{k}(s, \mathbf{z})$ for all $k \in \Theta$ and $\mathbf{z} \in \Omega$, where $\tilde{\boldsymbol{\varphi}}=\left[\tilde{\varphi}_{1}, \tilde{\varphi}_{2}, \ldots, \tilde{\varphi}_{n}\right]^{T} \in C_{F_{0}}\left[(-\infty, 0] \times \mathbb{R}^{m}, \mathbb{C}^{n}\right]$, in which $F_{0}=F_{s}$ on $(-\infty, 0] \times \mathbb{R}^{m}$ and $C_{F_{0}}\left[(-\infty, 0] \times \mathbb{R}^{m}, \mathbb{C}^{n}\right]$ are a family of all continuous complex-valued functions with a norm $\left\|\tilde{\varphi}_{k}(s, \mathbf{z})\right\|_{L^{2}}=\left(\int_{\Omega}\left|\tilde{\varphi}_{k}(s, \mathbf{z})\right|^{2} \mathrm{~d} \mathbf{z}\right)^{1 / 2}, k \in \Theta$.

Let $\tilde{u}_{k}(t, \mathbf{z})=\tilde{u}_{k}^{R}(t, \mathbf{z})+i \tilde{u}_{k}^{I}(t, \mathbf{z})$, and let the activation function $\tilde{g}_{j}\left(\tilde{u}_{j}(t, \mathbf{z})\right)$ be expressed by separating it into its real and imaginary parts as follows:

$$
\tilde{g}_{j}\left(\tilde{u}_{j}(t, \mathbf{z})\right)=\tilde{g}_{j}^{R}\left(\tilde{u}_{j}^{R}(t, \mathbf{z}), \tilde{u}_{j}^{I}(t, \mathbf{z})\right)+i \tilde{g}_{j}^{I}\left(\tilde{u}_{j}^{R}(t, \mathbf{z}), \tilde{u}_{j}^{I}(t, \mathbf{z})\right),
$$

where $\tilde{g}_{j}^{R}\left(\tilde{u}_{j}^{R}(t, \mathbf{z}), \tilde{u}_{j}^{I}(t, \mathbf{z})\right): \mathbb{R}^{2} \rightarrow \mathbb{R}, \tilde{g}_{j}^{I}\left(\tilde{u}_{j}^{R}(t, \mathbf{z}), \tilde{u}_{j}^{I}(t, \mathbf{z})\right): \mathbb{R}^{2} \rightarrow \mathbb{R}, k, j \in \Theta$.

Furthermore, we rewrite (1) by separating it into real and imaginary parts:

$$
\begin{aligned}
& \left\{\begin{aligned}
& \frac{\partial \tilde{u}_{k}^{R}(t, \mathbf{z})}{\partial t}= \pi_{k} \sum_{h=1}^{m} \frac{\partial}{\partial z_{h}}\left(\frac{\partial \tilde{u}_{k}^{R}(t, \mathbf{z})}{\partial z_{h}}\right)-d_{k} \tilde{u}_{k}^{R}(t, \mathbf{z}) \\
&+\sum_{j=1}^{n}\left[a_{k j}^{R} \tilde{g}_{j}^{R}\left(\tilde{u}_{j}^{R}(t, \mathbf{z}), \tilde{u}_{j}^{I}(t, \mathbf{z})\right)-a_{k j}^{I} \tilde{g}_{j}^{I}\left(\tilde{u}_{j}^{R}(t, \mathbf{z}), \tilde{u}_{j}^{I}(t, \mathbf{z})\right)\right] \\
&+\sum_{j=1}^{n}\left[b_{k j}^{R} \tilde{g}_{j}^{R}\left(\tilde{u}_{j}^{R}\left(t-\tau_{k j}(t), \mathbf{z}\right), \tilde{u}_{j}^{I}\left(t-\tau_{k j}(t), \mathbf{z}\right)\right)\right. \\
&\left.-b_{k j}^{I} \tilde{g}_{j}^{I}\left(\tilde{u}_{j}^{R}\left(t-\tau_{k j}(t), \mathbf{z}\right), \tilde{u}_{j}^{I}\left(t-\tau_{k j}(t), \mathbf{z}\right)\right)\right] \\
&+\sum_{j=1}^{n}\left[q_{k j}^{R} \int_{-\infty}^{t} \sigma_{k j}(t-s) \tilde{g}_{j}^{R}\left(\tilde{u}_{j}^{R}(s, \mathbf{z}), \tilde{u}_{j}^{I}(s, \mathbf{z})\right) \mathrm{d} s\right. \\
&\left.-q_{k j}^{I} \int_{-\infty}^{t} \sigma_{k j}(t-s) \tilde{g}_{j}^{I}\left(\tilde{u}_{j}^{R}(s, \mathbf{z}), \tilde{u}_{j}^{I}(s, \mathbf{z})\right) \mathrm{d} s\right]+J_{k}^{R} \\
&\left.\tilde{u}_{k}^{R}(t, \mathbf{z})\right|_{\mathbf{z} \in \partial \Omega}=0,
\end{aligned}\right. \\
& \left\{\begin{aligned}
& \frac{\partial \tilde{u}_{k}^{I}(t, \mathbf{z})}{\partial t}= \pi_{k} \sum_{h=1}^{m} \frac{\partial}{\partial z_{h}}\left(\frac{\partial \tilde{u}_{k}^{I}(t, \mathbf{z})}{\partial z_{h}}\right)-d_{k} \tilde{u}_{k}^{I}(t, \mathbf{z}) \\
&+\sum_{j=1}^{n}\left[a_{k j}^{R} \tilde{g}_{j}^{I}\left(\tilde{u}_{j}^{R}(t, \mathbf{z}), \tilde{u}_{j}^{I}(t, \mathbf{z})\right)+a_{k j}^{I} \tilde{g}_{j}^{R}\left(\tilde{u}_{j}^{R}(t, \mathbf{z}), \tilde{u}_{j}^{I}(t, \mathbf{z})\right)\right] \\
&+\sum_{j=1}^{n}\left[b_{k j}^{R} \tilde{g}_{j}^{I}\left(\tilde{u}_{j}^{R}\left(t-\tau_{k j}(t), \mathbf{z}\right), \tilde{u}_{j}^{I}\left(t-\tau_{k j}(t), \mathbf{z}\right)\right)\right. \\
&\left.+b_{k j}^{I} \tilde{g}_{j}^{R}\left(\tilde{u}_{j}^{R}\left(t-\tau_{k j}(t), \mathbf{z}\right), \tilde{u}_{j}^{I}\left(t-\tau_{k j}(t), \mathbf{z}\right)\right)\right] \\
&+\sum_{j=1}^{n}\left[q_{k j}^{R} \int_{-\infty}^{t} \sigma_{k j}(t-s) \tilde{g}_{j}^{I}\left(\tilde{u}_{j}^{R}(s, \mathbf{z}), \tilde{u}_{j}^{I}(s, \mathbf{z})\right) \mathrm{d} s\right. \\
&\left.+q_{k j}^{I} \int_{-\infty}^{t} \sigma_{k j}(t-s) \tilde{g}_{j}^{R}\left(\tilde{u}_{j}^{R}(s, \mathbf{z}), \tilde{u}_{j}^{I}(s, \mathbf{z})\right) \mathrm{d} s\right]+J_{k}^{I} \\
&\left.\tilde{u}_{k}^{I}(t, \mathbf{z})\right|_{\mathbf{z} \in \partial \Omega}=0,
\end{aligned}\right.
\end{aligned}
$$

where $k \in \Theta, \mathbf{A}^{R}=\left(a_{k j}^{R}\right)_{n \times n}, \mathbf{B}^{R}=\left(b_{k j}^{R}\right)_{n \times n}, \mathbf{Q}^{R}=\left(q_{k j}^{R}\right)_{n \times n}$, and $\mathbf{J}^{R}=\left(J_{1}^{R}, J_{2}^{R}, \ldots, J_{n}^{R}\right)^{T}$ are, respectively, the real parts of $\mathbf{A}, \mathbf{B}, \mathbf{Q}$, and $\mathbf{J} . \mathbf{A}^{I}=\left(a_{k j}^{I}\right)_{n \times n}, \mathbf{B}^{I}=\left(b_{k j}^{I}\right)_{n \times n}, \mathbf{Q}^{I}=\left(q_{k j}^{I}\right)_{n \times n}$, and $\mathbf{J}^{I}=\left(J_{1}^{I}, J_{2}^{I}, \ldots, J_{n}^{I}\right)^{T}$ are, respectively, the imaginary parts of $\mathbf{A}, \mathbf{B}, \mathbf{Q}$, and $\mathbf{J}$.

Let $\tilde{\mathbf{u}}^{\#}=\left(\tilde{u}_{1}^{\#}, \tilde{u}_{2}^{\#}, \ldots, \tilde{u}_{n}^{\#}\right)^{T}$ be the equilibrium state of (1), where $\tilde{u}_{k}^{\#}=\tilde{u}_{k}^{\# R}+i \tilde{u}_{k}^{\# I}, k \in \Theta$.

Assumption 1 Supposing that $\tau_{k j}(t) \geq 0(k, j \in \Theta)$ is the time-varying delays of (1) from the $k$ th neuron to the $j$ th neuron. Let $\tau=\max _{k, j \in \Theta} \sup _{t \geq 0}\left\{\tau_{k j}(t)\right\}>0$.

Remark 1 In [9, 15-19, 21, 24, 25], time-varying delays were considered in the addressed NNs. Let $\tau_{k j}(t)$ be a differential with a bounded derivative because the scalar Lyapunov function method is adopted in $[9,15-19,21,24,25]$ to analyze the stability of the systems, which are constructed inevitably with time-varying delay terms. We introduced $\tau_{k j}(t)$ into 
the CVNNs in $[5,20]$ and applied the vector Lyapunov function method to study the exponential stability of the equilibrium point of the systems. The candidate vector Lyapunov function with no delay terms included has a very simple form, which makes the existence of $\dot{\tau}_{k j}(t)$ with boundedness (i.e. $0 \leq \dot{\tau}_{k j}(t)<\sigma<1, k, j \in \Theta$ ) is no longer necessary. Therefore, Assumption 1 is more general than the ones in [9, 15-19, 21, 24, 25].

Let $\mathbf{z}=\left(z_{1}, z_{2}, \ldots, z_{m}\right)^{\mathrm{T}} \in \Omega$.

Assumption 2 It is assumed that $\left|z_{h}\right| \leq \omega_{h}$, where $\mathbf{z} \in \Omega$ and $h=1,2, \ldots, m$.

Assumption 3 Assuming that $\sigma_{k j}:[0, \infty) \rightarrow[0, \infty)$ is the delay kernels from the $k$ th neuron to the $j$ th neuron, which is a piecewise continuous function that satisfies the following equation:

$$
\int_{0}^{\infty} e^{\beta s} \sigma_{k j}(s) \mathrm{d} s=\rho_{k j}(\beta), \quad k, j \in \Theta
$$

where $\rho_{k j}(\beta)$ with $\rho_{k j}(0)=1$ is a continuous function on $[0, \delta]$.

Assumption 4 Suppose that $\tilde{g}_{j}(\cdot)$ with the form of (2) satisfies the following conditions:

(a) The partial derivatives $\tilde{g}_{j}(\cdot)$ with respect to $\tilde{u}_{j}^{R}$ and $\tilde{u}_{j}^{I}: \partial \tilde{g}_{j}^{R} / \partial \tilde{u}_{j}^{R}, \partial \tilde{g}_{j}^{R} / \partial \tilde{u}_{j}^{I}, \partial \tilde{g}_{j}^{I} / \partial \tilde{u}_{j}^{R}$, and $\partial \tilde{g}_{j}^{I} / \partial \tilde{u}_{j}^{I}$ exist and are continuous.

(b) The partial derivatives $\partial \tilde{g}_{j}^{R} / \partial \tilde{u}_{j}^{R}, \partial \tilde{g}_{j}^{R} / \partial \tilde{u}_{j}^{I}, \partial \tilde{g}_{j}^{I} / \partial \tilde{u}_{j}^{R}$, and $\partial \tilde{g}_{j}^{I} / \partial \tilde{u}_{j}^{I}$ are bounded, i.e. there exist positive constants $l_{j}^{R R}, l_{j}^{R I}, l_{j}^{I R}$, and $l_{j}^{I I}$ such that $\left|\partial \tilde{g}_{j}^{R} / \partial \tilde{u}_{j}^{R}\right| \leq l_{j}^{R R},\left|\partial \tilde{g}_{j}^{R} / \partial \tilde{u}_{j}^{I}\right| \leq l_{j}^{R I}$, $\left|\partial \tilde{g}_{j}^{I} / \partial \tilde{u}_{j}^{R}\right| \leq l_{j}^{I R}$, and $\left|\partial \tilde{g}_{j}^{I} / \partial \tilde{u}_{j}^{I}\right| \leq l_{j}^{I I}$. Then, according to the mean value theorem of multivariable functions, for any $\tilde{u}_{j}^{R}, \hat{u}_{j}^{R}, \tilde{u}_{j}^{I}, \hat{u}_{j}^{I} \in \mathbb{R}$, and $j \in \Theta$, we have

$$
\begin{aligned}
& \left|\tilde{g}_{j}^{R}\left(\tilde{u}_{j}^{R}, \tilde{u}_{j}^{I}\right)-\tilde{g}_{j}^{R}\left(\hat{u}_{j}^{R}, \hat{u}_{j}^{I}\right)\right| \leq l_{j}^{R R}\left|\tilde{u}_{j}^{R}-\hat{u}_{j}^{R}\right|+l_{j}^{R I}\left|\tilde{u}_{j}^{I}-\hat{u}_{j}^{I}\right|, \\
& \left|\tilde{g}_{j}^{I}\left(\tilde{u}_{j}^{R}, \tilde{u}_{j}^{I}\right)-\tilde{g}_{j}^{I}\left(\hat{u}_{j}^{R}, \hat{u}_{j}^{I}\right)\right| \leq l_{j}^{I R}\left|\tilde{u}_{j}^{R}-\hat{u}_{j}^{R}\right|+l_{j}^{I I}\left|\tilde{u}_{j}^{I}-\hat{u}_{j}^{I}\right| .
\end{aligned}
$$

Let $\mathbf{L}^{R R}=\operatorname{diag}\left(l_{1}^{R R}, l_{2}^{R R}, \ldots, l_{n}^{R R}\right), \mathbf{L}^{R I}=\operatorname{diag}\left(l_{1}^{R I}, l_{2}^{R I}, \ldots, l_{n}^{R I}\right), \mathbf{L}^{I R}=\operatorname{diag}\left(l_{1}^{I R}, l_{2}^{I R}, \ldots, l_{n}^{I R}\right)$, and $\mathbf{L}^{I I}=\operatorname{diag}\left(l_{1}^{I I}, l_{2}^{I I}, \ldots, l_{n}^{I I}\right)$.

Definition 1 The equilibrium state $\tilde{\mathbf{u}}^{\#}$ of (1) is globally exponentially stable if there exist constants $\Gamma>0$ and $\lambda>0$ such that, for all $\mathbf{z} \in \Omega, \mathbf{J} \in \mathbb{C}^{n}$, and $t \geq 0$, the following inequality holds:

$$
\left\|\tilde{\mathbf{u}}-\tilde{\mathbf{u}}^{\#}\right\|_{L^{2}} \leq \sup _{s \in(-\infty, 0]}\left\|\tilde{\boldsymbol{\varphi}}(s, \mathbf{z})-\tilde{\mathbf{u}}^{\#}\right\|_{L^{2}} \Gamma e^{-\lambda t} .
$$

Definition 2 Let $\mathbf{X}$ and $\mathbf{Y}$ be topological space. If the mapping $f: \mathbf{X} \rightarrow \mathbf{Y}$ satisfies the following conditions, then $f$ is called homeomorphism:

(i) $f$ is injective from $\mathbf{X}$ to $\mathbf{Y}$;

(ii) $f$ is surjective from $\mathbf{X}$ to $\mathbf{Y}$;

(iii) the inverse mapping $f^{-1}$ is existed and continuous.

Lemma 1 ([5]) Assuming that $\mathbf{B}=\left(b_{k j}\right)_{n \times n} \in \mathbb{R}^{n \times n}$ is a matrix with $b_{k j} \leq 0(k \neq j, k, j \in \Theta)$, the following statements are equivalent: 
(a) The matrix $\mathbf{B}$ is an M-matrix.

(b) The real parts of all eigenvalues of $\mathbf{B}$ are positive.

(c) There exists a positive vector $\mathbf{5} \in \mathbb{R}^{n}$ such that $\mathbf{B} \boldsymbol{\varsigma}>\mathbf{0}$.

Lemma 2 ([5]) Assuming that $\mathbf{H}(\tilde{\mathbf{u}})$ is a continuous function, which is defined on $\mathbb{R}^{n}$, if $\mathbf{H}(\tilde{\mathbf{u}})$ is injective on $\mathbb{R}^{n}$ and satisfies $\lim _{\|\tilde{\mathbf{u}}\| \rightarrow \infty}\|\mathbf{H}(\tilde{\mathbf{u}})\| \rightarrow \infty$, then $\mathbf{H}(\tilde{\mathbf{u}})$ is a homeomorphism of $\mathbb{R}^{n}$ into itself.

Lemma 3 ([30]) Let $\Omega \in \mathbb{R}^{m}$ be a compact set with a smooth boundary $\partial \Omega$. For all $\mathbf{z} \in \Omega$, let $\left|z_{h}\right| \leq \omega_{h} \leq \hat{\omega}(h=1,2, \ldots, m)$ and let $u(\mathbf{z}) \in \mathbb{R}$ be a real-valued function belonging to $\mathrm{C}^{1}(\Omega)$ which vanish on the boundary $\partial \Omega$ of $\Omega$. Then $\int_{\Omega}|u(\mathbf{z})|^{2} \mathrm{~d} \mathbf{z} \leq \frac{\hat{\omega}^{2}}{m} \int_{\Omega} \nabla u^{\mathrm{T}}(\mathbf{z}) \nabla u(\mathbf{z}) \mathrm{d} \mathbf{z}$, here $\nabla(u(\mathbf{z}))=\left(\frac{\partial u(\mathbf{z})}{\partial z_{1}}, \frac{\partial u(\mathbf{z})}{\partial z_{2}}, \ldots, \frac{\partial u(\mathbf{z})}{\partial z_{m}}\right)^{\mathrm{T}}$.

Proof If there exists some $h^{\prime} \in\{1,2, \ldots, m\}$ such that $\int_{-\omega_{h^{\prime}}}^{\omega_{h^{\prime}}}|u(\mathbf{z})|^{2} \mathrm{~d} z_{h^{\prime}}=0$, the result is obvious.

Next, we consider that $\int_{-\omega_{h}}^{\omega_{h}}|u(\mathbf{z})|^{2} \mathrm{~d} z_{h} \neq 0$ for any $h \in\{1,2, \ldots, m\}$.

For any $\mathbf{z} \in \Omega$ and each fixed $h \in\{1,2, \ldots, m\}$, we have

$$
u(\mathbf{z})=\int_{-\omega_{h}}^{z_{h}} \frac{\partial u(\mathbf{z})}{\partial z_{h}} \mathrm{~d} z_{h}, \quad u(\mathbf{z})=-\int_{z_{h}}^{\omega_{h}} \frac{\partial u(\mathbf{z})}{\partial z_{h}} \mathrm{~d} z_{h} .
$$

It can be concluded that $2|u(\mathbf{z})| \leq \int_{-\omega_{h}}^{\omega_{h}}\left|\frac{\partial u(\mathbf{z})}{\partial z_{h}}\right| \mathrm{d} z_{h}, h \in\{1,2, \ldots, m\}$.

Using Schwarz's inequality, we obtain $4|u(\mathbf{z})|^{2} \leq 2 \omega_{h} \int_{-\omega_{h}}^{\omega_{h}}\left|\frac{\partial u(z)}{\partial z_{h}}\right|^{2} \mathrm{~d} z_{h}$.

Integrating both sides of the above inequality with respect to $\mathbf{z}$ over the domain $\Omega$ gives the following equation:

$$
\int_{\Omega} 4|u(\mathbf{z})|^{2} \mathrm{~d} \mathbf{z} \leq \int_{\Omega} 2 \omega_{h}\left(\int_{-\omega_{h}}^{\omega_{h}}\left|\frac{\partial u(\mathbf{z})}{\partial z_{h}}\right|^{2} \mathrm{~d} z_{h}\right) \mathrm{d} \mathbf{z}=4 \omega_{h}^{2} \int_{\Omega}\left|\frac{\partial u(\mathbf{z})}{\partial z_{h}}\right|^{2} \mathrm{~d} \mathbf{z},
$$

i.e. $\int_{\Omega}|u(\mathbf{z})|^{2} \mathrm{~d} \mathbf{z} \leq \omega_{h}^{2} \int_{\Omega}\left|\frac{\partial u(\mathbf{z})}{\partial z_{h}}\right|^{2} \mathrm{~d} \mathbf{z}, h \in\{1,2, \ldots, m\}$.

The following inequalities hold:

$$
\int_{\Omega}|u(\mathbf{z})|^{2} \mathrm{~d} \mathbf{z} \leq \frac{\hat{\omega}^{2}}{m} \int_{\Omega} \nabla u^{\mathrm{T}}(\mathbf{z}) \nabla u(\mathbf{z}) \mathrm{d} \mathbf{z} .
$$

Therefore, the proof is completed.

In $[32,36,37,40]$, the lemmas have been provided to deal with the reaction-diffusion term of NNs. To make a convenient comparison with Lemma 3, we adopt the same notation definitions for the lemmas described in [32, 36, 37, 40].

Lemma $4([32,36,37,40])$ Let $\Omega \in \mathbb{R}^{m}$ be a compact set with a smooth boundary $\partial \Omega$. For all $\mathbf{z} \in \Omega$, let $\left|z_{h}\right| \leq \omega_{h} \leq \hat{\omega}(h=1,2, \ldots, m)$, and let $u(\mathbf{z}) \in \mathbb{R}$ be a real-valued function belonging to $\mathrm{C}^{1}(\Omega)$ which vanish on the boundary $\partial \Omega$ of $\Omega$. Then $\int_{\Omega}|u(\mathbf{z})|^{2} \mathrm{~d} \mathbf{z} \leq$ $\hat{\omega}^{2} \int_{\Omega} \nabla u^{\mathrm{T}}(\mathbf{z}) \nabla u(\mathbf{z}) \mathrm{d} \mathbf{z}$.

Remark 2 Note that when $p=2$, Lemma 2.2 [36] and Lemma 2 [40] are the same as Lemma 2.2 [32] and Lemma 2.1 [37]. By comparison, we find that the difference between Lemma 
3 and Lemma 4 is a coefficient $m$ that is the dimension of the space $\Omega$. From the conditions obtained to ensure the stability of the addressed systems in [32, 36, 37, 40], we can see that the reaction-diffusion terms have a positive effect on the stability of the NNs. $\int_{\Omega}|u(\mathbf{z})|^{2} \mathrm{~d} \mathbf{z} \leq \frac{\hat{\omega}^{2}}{m} \int_{\Omega} \nabla u^{\mathrm{T}}(\mathbf{z}) \nabla u(\mathbf{z}) \mathrm{d} \mathbf{z}$ in Lemma 3 has a strong positive influence on stability compared to Lemma 4.

\section{Main results}

For simplification, let

$$
\begin{aligned}
& \widehat{\Theta}=\{1,2, \ldots, n, n+1, n+2, \ldots, 2 n\}, \\
& \tilde{\mathbf{u}}^{R}=\left(\tilde{u}_{1}^{R}, \tilde{u}_{2}^{R}, \ldots, \tilde{u}_{n}^{R}\right)^{\mathrm{T}}, \quad \tilde{\mathbf{u}}^{I}=\left(\tilde{u}_{1}^{I}, \tilde{u}_{2}^{I}, \ldots, \tilde{u}_{n}^{I}\right)^{\mathrm{T}}, \\
& \tilde{\boldsymbol{\alpha}}=\left(\left(\tilde{\mathbf{u}}^{R}\right)^{\mathrm{T}},\left(\tilde{\mathbf{u}}^{I}\right)^{\mathrm{T}}\right)^{\mathrm{T}}, \quad \tilde{\mathbf{J}}=\left(\left(\tilde{\mathbf{J}}^{R}\right)^{\mathrm{T}},\left(\tilde{\mathbf{J}}^{I}\right)^{\mathrm{T}}\right)^{\mathrm{T}}, \\
& \tilde{\mathbf{G}}^{R}(\tilde{\boldsymbol{\alpha}})=\left(\left(\tilde{\mathbf{g}}^{R}\left(\tilde{\mathbf{u}}^{R}, \tilde{\mathbf{u}}^{I}\right)\right)^{\mathrm{T}},\left(\tilde{\mathbf{g}}^{R}\left(\tilde{\mathbf{u}}^{R}, \tilde{\mathbf{u}}^{I}\right)\right)^{\mathrm{T}}\right)^{\mathrm{T}}, \quad \tilde{\mathbf{G}}^{I}(\tilde{\boldsymbol{\alpha}})=\left(\left(\tilde{\mathbf{g}}^{I}\left(\tilde{\mathbf{u}}^{R}, \tilde{\mathbf{u}}^{I}\right)\right)^{\mathrm{T}},\left(\tilde{\mathbf{g}}^{I}\left(\tilde{\mathbf{u}}^{R}, \tilde{\mathbf{u}}^{I}\right)\right)^{\mathrm{T}}\right)^{\mathrm{T}} .
\end{aligned}
$$

Define a map associated with (3) and (4) as follows:

$$
\mathbf{H}(\tilde{\boldsymbol{\alpha}})=\tilde{\boldsymbol{\pi}} \nabla(\nabla \tilde{\boldsymbol{\alpha}}) \mathbf{I}^{\prime}-\tilde{\mathbf{D}} \tilde{\boldsymbol{\alpha}}+\mathbf{P}_{1} \tilde{\mathbf{G}}^{R}(\tilde{\boldsymbol{\alpha}})+\mathbf{P}_{2} \tilde{\mathbf{G}}^{I}(\tilde{\boldsymbol{\alpha}})+\tilde{\mathbf{J}}
$$

where $\mathbf{I}^{\prime} \in \mathbb{R}^{m}$ is an unit vector, and

$$
\begin{aligned}
& \tilde{\boldsymbol{\pi}}=\left[\begin{array}{ll}
\boldsymbol{\pi} & \mathbf{0} \\
\mathbf{0} & \boldsymbol{\pi}
\end{array}\right], \quad \tilde{\mathbf{D}}=\left[\begin{array}{ll}
\mathbf{D} & \mathbf{0} \\
\mathbf{0} & \mathbf{D}
\end{array}\right], \quad \mathbf{P}_{1}=\left[\begin{array}{cc}
\mathbf{A}^{R}+\mathbf{B}^{R}+\mathbf{Q}^{R} & \mathbf{0} \\
\mathbf{0} & \mathbf{A}^{I}+\mathbf{B}^{I}+\mathbf{Q}^{I}
\end{array}\right], \\
& \mathbf{P}_{2}=\left[\begin{array}{cc}
-\mathbf{A}^{I}-\mathbf{B}^{I}-\mathbf{Q}^{I} & \mathbf{0} \\
\mathbf{0} & \mathbf{A}^{R}+\mathbf{B}^{R}+\mathbf{Q}^{R}
\end{array}\right] .
\end{aligned}
$$

According to Definition 2, we know that, if $\mathbf{H}(\tilde{\boldsymbol{\alpha}})$ is a homeomorphism on $\mathbb{R}^{2 n}$, (1) has a unique equilibrium state $\tilde{\mathbf{u}}^{\#}$. Next, a theorem will be given to assure that $\mathbf{H}(\tilde{\boldsymbol{\alpha}})$ is a homeomorphism on $\mathbb{R}^{2 n}$.

Theorem 1 Suppose that Assumptions 1-4 are satisfied. Then (1) has a unique equilibrium state for all $\mathbf{J} \in \mathbb{C}^{n}$ and $\mathbf{z} \in \Omega$ if $\tilde{\mathbf{T}}-\tilde{\mathbf{P}} \tilde{\mathbf{L}}$ is an M-matrix, where $\tilde{\mathbf{T}}=\operatorname{diag}\left(\tilde{T}_{k}\right)_{2 n \times 2 n}=$ $\left[\begin{array}{ll}\mathbf{T} & 0 \\ 0 & \mathrm{~T}\end{array}\right], \mathbf{T}=\operatorname{diag}\left(T_{k}\right)_{n \times n}$ with

$$
\begin{aligned}
T_{k}= & 2 \frac{m \pi_{k}}{\hat{\omega}^{2}}+2 d_{k}-\sum_{j=1}^{n}\left[\left(l_{j}^{R R}+l_{j}^{R I}\right)\left(\left|a_{k j}^{R}\right|+\left|b_{k j}^{R}\right|+\left|q_{k j}^{R}\right|\right)\right. \\
& \left.+\left(l_{j}^{I R}+l_{j}^{I I}\right)\left(\left|a_{k j}^{I}\right|+\left|b_{k j}^{I}\right|+\left|q_{k j}^{I}\right|\right)\right], \quad k \in \Theta,
\end{aligned}
$$

and

$$
\tilde{\mathbf{P}}=\left[\begin{array}{cc}
\left|\mathbf{A}^{R}\right|+\left|\mathbf{B}^{R}\right|+\left|\mathbf{Q}^{R}\right| & \left|\mathbf{A}^{I}\right|+\left|\mathbf{B}^{I}\right|+\left|\mathbf{Q}^{I}\right| \\
\left|\mathbf{A}^{I}\right|+\left|\mathbf{B}^{I}\right|+\left|\mathbf{Q}^{I}\right| & \left|\mathbf{A}^{R}\right|+\left|\mathbf{B}^{R}\right|+\left|\mathbf{Q}^{R}\right|
\end{array}\right], \quad \tilde{\mathbf{L}}=\left[\begin{array}{cc}
\mathbf{L}^{R R} & \mathbf{L}^{R I} \\
\mathbf{L}^{I R} & \mathbf{L}^{I I}
\end{array}\right]
$$

Proof (i) First, the map $\mathbf{H}(\tilde{\boldsymbol{\alpha}})$ is proven to be injective. 
It is assumed that there exist $\tilde{\boldsymbol{\alpha}}, \tilde{\boldsymbol{\alpha}^{\prime}} \in \mathbb{R}^{2 n}$ with $\tilde{\boldsymbol{\alpha}} \neq \tilde{\boldsymbol{\alpha}}^{\prime}$ such that $\mathbf{H}(\tilde{\boldsymbol{\alpha}})=\mathbf{H}\left(\tilde{\boldsymbol{\alpha}}^{\prime}\right)$, i.e.

$$
\tilde{\boldsymbol{\pi}} \nabla(\nabla \tilde{\boldsymbol{\alpha}}) \mathbf{I}^{\prime}-\tilde{\mathbf{D}} \tilde{\boldsymbol{\alpha}}+\mathbf{P}_{1} \tilde{\mathbf{G}}^{R}(\tilde{\boldsymbol{\alpha}})+\mathbf{P}_{2} \tilde{\mathbf{G}}^{I}(\tilde{\boldsymbol{\alpha}})=\tilde{\boldsymbol{\pi}} \nabla\left(\nabla \tilde{\boldsymbol{\alpha}}^{\prime}\right) \mathbf{I}^{\prime}-\tilde{\mathbf{D}} \tilde{\boldsymbol{\alpha}}^{\prime}+\mathbf{P}_{1} \tilde{\mathbf{G}}^{R}\left(\tilde{\boldsymbol{\alpha}}^{\prime}\right)+\mathbf{P}_{2} \tilde{\mathbf{G}}^{I}\left(\tilde{\boldsymbol{\alpha}}^{\prime}\right),
$$

which means

$$
\tilde{\mathbf{D}}\left(\tilde{\boldsymbol{\alpha}}-\tilde{\boldsymbol{\alpha}}^{\prime}\right)=\tilde{\boldsymbol{\pi}}\left[\nabla(\nabla \tilde{\boldsymbol{\alpha}})-\nabla\left(\nabla \tilde{\boldsymbol{\alpha}}^{\prime}\right)\right] \mathbf{I}^{\prime}+\mathbf{P}_{1}\left[\tilde{\mathbf{G}}^{R}(\tilde{\boldsymbol{\alpha}})-\tilde{\mathbf{G}}^{R}\left(\tilde{\boldsymbol{\alpha}}^{\prime}\right)\right]+\mathbf{P}_{2}\left[\tilde{\mathbf{G}}^{I}(\tilde{\boldsymbol{\alpha}})-\tilde{\mathbf{G}}^{I}\left(\tilde{\boldsymbol{\alpha}}^{\prime}\right)\right]
$$

or

$$
\begin{aligned}
& d_{k}\left(\tilde{u}_{k}^{R}-\tilde{u}_{k}^{\prime R}\right) \\
& =\pi_{k} \sum_{h=1}^{m} \frac{\partial}{\partial z_{h}}\left(\frac{\partial \tilde{u}_{k}^{R}}{\partial z_{h}}-\frac{\partial \tilde{u}_{k}^{\prime R}}{\partial z_{h}}\right)+\sum_{j=1}^{n}\left(a_{k j}^{R}+b_{k j}^{R}+q_{k j}^{R}\right)\left[\tilde{g}_{j}^{R}\left(\tilde{u}_{j}^{R}, \tilde{u}_{j}^{I}\right)-\tilde{g}_{j}^{R}\left(\tilde{u}_{j}^{\prime R}, \tilde{u}_{j}^{\prime I}\right)\right] \\
& \quad+\sum_{j=1}^{n}\left(a_{k j}^{I}+b_{k j}^{I}+q_{k j}^{I}\right)\left[\tilde{g}_{j}^{I}\left(\tilde{u}_{j}^{R}, \tilde{u}_{j}^{I}\right)-\tilde{g}_{j}^{I}\left(\tilde{u}_{j}^{\prime R}, \tilde{u}_{j}^{\prime I}\right)\right], \\
& d_{k}\left(\tilde{u}_{k}^{I}-\tilde{u}_{k}^{\prime I}\right) \\
& =\pi_{k} \sum_{h=1}^{m} \frac{\partial}{\partial z_{h}}\left(\frac{\partial \tilde{u}_{k}^{I}}{\partial z_{h}}-\frac{\partial \tilde{u}_{k}^{\prime I}}{\partial z_{h}}\right)+\sum_{j=1}^{n}\left(a_{k j}^{R}+b_{k j}^{R}+q_{k j}^{R}\right)\left[\tilde{g}_{j}^{I}\left(\tilde{u}_{j}^{R}, \tilde{u}_{j}^{I}\right)-\tilde{g}_{j}^{I}\left(\tilde{u}_{j}^{\prime R}, \tilde{u}_{j}^{\prime I}\right)\right] \\
& \quad+\sum_{j=1}^{n}\left(a_{k j}^{I}+b_{k j}^{I}+q_{k j}^{I}\right)\left[\tilde{g}_{j}^{R}\left(\tilde{u}_{j}^{R}, \tilde{u}_{j}^{I}\right)-\tilde{g}_{j}^{R}\left(\tilde{u}_{j}^{\prime R}, \tilde{u}_{j}^{\prime I}\right)\right] .
\end{aligned}
$$

Multiplying by $\tilde{u}_{k}^{R}-\tilde{u}_{k}^{\prime R}$ on both sides of (9), then integrating on both sides on the domain $\Omega$ and considering Assumptions 1-4 and Lemma 3, we obtain

$$
\begin{aligned}
\int_{\Omega} & d_{k}\left(\tilde{u}_{k}^{R}-\tilde{u}_{k}^{\prime R}\right)^{2} \mathrm{~d} \mathbf{z} \\
= & \pi_{k} \int_{\Omega} \sum_{h=1}^{m}\left(\tilde{u}_{k}^{R}-\tilde{u}_{k}^{\prime R}\right) \frac{\partial}{\partial z_{h}}\left(\frac{\partial \tilde{u}_{k}^{R}}{\partial z_{h}}-\frac{\partial \tilde{u}_{k}^{\prime R}}{\partial z_{h}}\right) \mathrm{d} \mathbf{z} \\
& +\int_{\Omega}\left(\tilde{u}_{k}^{R}-\tilde{u}_{k}^{\prime R}\right) \sum_{j=1}^{n}\left(a_{k j}^{R}+b_{k j}^{R}+q_{k j}^{R}\right)\left[\tilde{g}_{j}^{R}\left(\tilde{u}_{j}^{R}, \tilde{u}_{j}^{I}\right)-\tilde{g}_{j}^{R}\left(\tilde{u}_{j}^{\prime R}, \tilde{u}_{j}^{\prime I}\right)\right] \mathrm{d} \mathbf{z} \\
& +\int_{\Omega}\left(\tilde{u}_{k}^{R}-\tilde{u}_{k}^{\prime R}\right) \sum_{j=1}^{n}\left[a_{k j}^{I}+b_{k j}^{I}+q_{k j}^{I}\right]\left[\tilde{g}_{j}^{I}\left(\tilde{u}_{j}^{R}, \tilde{u}_{j}^{I}\right)-\tilde{g}_{j}^{I}\left(\tilde{u}_{j}^{\prime R}, \tilde{u}_{j}^{\prime I}\right)\right] \mathrm{d} \mathbf{z} \\
\leq & -\frac{m \pi_{k}}{\hat{\omega}^{2}} \int_{\Omega}\left(\tilde{u}_{k}^{R}-\tilde{u}_{k}^{\prime R}\right)^{2} \mathrm{~d} \mathbf{z} \\
& +\int_{\Omega}\left|\tilde{u}_{k}^{R}-\tilde{u}_{k}^{\prime R}\right| \sum_{j=1}^{n}\left[\left|a_{k j}^{R}\right|+\left|b_{k j}^{R}\right|+\left|q_{k j}^{R}\right|\right]\left[l_{j}^{R R}\left|\tilde{u}_{j}^{R}-\tilde{u}_{j}^{\prime R}\right|+l_{j}^{R I}\left|\tilde{u}_{j}^{I}-\tilde{u}_{j}^{\prime I}\right|\right] \mathrm{d} \mathbf{z} \\
& +\int_{\Omega}\left|\tilde{u}_{k}^{R}-\tilde{u}_{k}^{\prime R}\right| \sum_{j=1}^{n}\left[\left|a_{k j}^{I}\right|+\left|b_{k j}^{I}\right|+\left|q_{k j}^{I}\right|\right]\left[l_{j}^{I R}\left|\tilde{u}_{j}^{R}-\tilde{u}_{j}^{\prime R}\right|+l_{j}^{I I}\left|\tilde{u}_{j}^{I}-\tilde{u}_{j}^{\prime I}\right|\right] \mathrm{d} \mathbf{z} \\
\leq & \left\{-\frac{m \pi_{k}}{\hat{\omega}^{2}}+0.5 \sum_{j=1}^{n}\left[\left(\left|a_{k j}^{R}\right|+\left|b_{k j}^{R}\right|+\left|q_{k j}^{R}\right|\right)\left(l_{j}^{R R}+l_{j}^{R I}\right)\right.\right.
\end{aligned}
$$




$$
\begin{aligned}
& \left.\left.+\left(\left|a_{k j}^{I}\right|+\left|b_{k j}^{I}\right|+\left|q_{k j}^{I}\right|\right)\left(l_{j}^{I R}+l_{j}^{I I}\right)\right]\right\} \\
& \times \int_{\Omega}\left(\tilde{u}_{k}^{R}-\tilde{u}_{k}^{\prime R}\right)^{2} \mathrm{~d} \mathbf{z}+0.5 \sum_{j=1}^{n}\left[\left(\left|a_{k j}^{R}\right|+\left|b_{k j}^{R}\right|+\left|q_{k j}^{R}\right|\right) l_{j}^{R R}+\left(\left|a_{k j}^{I}\right|+\left|b_{k j}^{I}\right|+\left|q_{k j}^{I}\right|\right) l_{j}^{I R}\right] \\
& \times \int_{\Omega}\left(\tilde{u}_{j}^{R}-\tilde{u}_{j}^{\prime R}\right)^{2} \mathrm{~d} \mathbf{z} \\
& +0.5 \sum_{j=1}^{n}\left[\left(\left|a_{k j}^{R}\right|+\left|b_{k j}^{R}\right|+\left|q_{k j}^{R}\right|\right) l_{j}^{R I}+\left(\left|a_{k j}^{I}\right|+\left|b_{k j}^{I}\right|+\left|q_{k j}^{I}\right|\right) l_{j}^{I I}\right] \int_{\Omega}\left(\tilde{u}_{j}^{I}-\tilde{u}_{j}^{\prime I}\right)^{2} \mathrm{dz} .
\end{aligned}
$$

Multiplying by $\tilde{u}_{k}^{I}-\tilde{u}_{k}^{\prime I}$ on both sides of (10), then integrating on both sides on the domain $\Omega$, and considering Assumptions 1-4 and Lemma 3, we obtain

$$
\begin{aligned}
& d_{k} \int_{\Omega}\left(\tilde{u}_{k}^{I}-\tilde{u}_{k}^{\prime I}\right)^{2} \mathrm{~d} \mathbf{z} \\
& =\pi_{k} \int_{\Omega} \sum_{h=1}^{m}\left(\tilde{u}_{k}^{I}-\tilde{u}_{k}^{\prime I}\right) \frac{\partial}{\partial z_{h}}\left(\frac{\partial \tilde{u}_{k}^{I}}{\partial z_{h}}-\frac{\partial \tilde{u}_{k}^{\prime I}}{\partial z_{h}}\right) \mathrm{d} \mathbf{z} \\
& +\int_{\Omega}\left(\tilde{u}_{k}^{I}-\tilde{u}_{k}^{I I}\right) \sum_{j=1}^{n}\left(a_{k j}^{R}+b_{k j}^{R}+q_{k j}^{R}\right)\left[\tilde{g}_{j}^{I}\left(\tilde{u}_{j}^{R}, \tilde{u}_{j}^{I}\right)-\tilde{g}_{j}^{I}\left(\tilde{u}_{j}^{\prime R}, \tilde{u}_{j}^{\prime I}\right)\right] \mathrm{d} \mathbf{z} \\
& +\int_{\Omega}\left(\tilde{u}_{k}^{I}-\tilde{u}_{k}^{\prime I}\right) \sum_{j=1}^{n}\left(a_{k j}^{I}+b_{k j}^{I}+q_{k j}^{I}\right)\left[\tilde{g}_{j}^{R}\left(\tilde{u}_{j}^{R}, \tilde{u}_{j}^{I}\right)-\tilde{g}_{j}^{R}\left(\tilde{u}_{j}^{\prime R}, \tilde{u}_{j}^{\prime I}\right)\right] \mathrm{d} \mathbf{z} \\
& \leq-\int_{\Omega} \frac{m \pi_{k}}{\hat{\omega}^{2}}\left(\tilde{u}_{k}^{I}-\tilde{u}_{k}^{\prime I}\right)^{2} \mathrm{~d} \mathbf{z} \\
& \times \int_{\Omega}\left|\tilde{u}_{k}^{I}-\tilde{u}_{k}^{I I}\right| \sum_{j=1}^{n}\left(\left|a_{k j}^{R}\right|+\left|b_{k j}^{R}\right|+\left|q_{k j}^{R}\right|\right)\left(l_{j}^{I R}\left|\tilde{u}_{j}^{R}\right|+l_{j}^{I I}\left|\tilde{u}_{j}^{I}\right|\right) \mathrm{d} \mathbf{z} \\
& +\int_{\Omega}\left|\tilde{u}_{k}^{I}-\tilde{u}_{k}^{I I}\right| \sum_{j=1}^{n}\left(\left|a_{k j}^{I}\right|+\left|b_{k j}^{I}\right|+\left|q_{k j}^{I}\right|\right)\left(l_{j}^{R R}\left|\tilde{u}_{j}^{R}\right|+l_{j}^{R I}\left|\tilde{u}_{j}^{I}\right|\right) \mathrm{d} \mathbf{z} \\
& \leq\left\{-\frac{m \pi_{k}}{\hat{\omega}^{2}}+0.5 \sum_{j=1}^{n}\left[\left(\left|a_{k j}^{R}\right|+\left|b_{k j}^{R}\right|+\left|q_{k j}^{R}\right|\right)\left(l_{j}^{I R}+l_{j}^{I I}\right)\right.\right. \\
& \left.\left.+\left(\left|a_{k j}^{I}\right|+\left|b_{k j}^{I}\right|+\left|q_{k j}^{I}\right|\right)\left(l_{j}^{R R}+l_{j}^{R I}\right)\right]\right\} \\
& \times \int_{\Omega}\left(\tilde{u}_{k}^{I}-\tilde{u}_{k}^{\prime I}\right)^{2} \mathrm{~d} \mathbf{z}+0.5 \sum_{j=1}^{n}\left[\left(\left|a_{k j}^{I}\right|+\left|b_{k j}^{I}\right|+\left|q_{k j}^{I}\right|\right) l_{j}^{R R}\right. \\
& \left.+\left(\left|a_{k j}^{R}\right|+\left|b_{k j}^{R}\right|+\left|q_{k j}^{R}\right|\right) l_{j}^{I R}\right] \int_{\Omega}\left(\tilde{u}_{j}^{R}-\tilde{u}_{j}^{\prime R}\right)^{2} \mathrm{~d} \mathbf{z} \\
& +0.5 \sum_{j=1}^{n}\left[\left(\left|a_{k j}^{R}\right|+\left|b_{k j}^{R}\right|+\left|q_{k j}^{R}\right|\right) l_{j}^{I I}+\left(\left|a_{k j}^{I}\right|+\left|b_{k j}^{I}\right|+\left|q_{k j}^{I}\right|\right) l_{j}^{R I}\right] \int_{\Omega}\left(\tilde{u}_{j}^{I}-\tilde{u}_{j}^{\prime I}\right)^{2} \mathrm{~d} \mathbf{z} .
\end{aligned}
$$

The inequalities (11) and (12) are rewritten as follows:

$$
\mathbf{T}\left\|\tilde{\mathbf{u}}^{R}-\tilde{\mathbf{u}}^{\prime R}\right\|_{L^{2}}^{2}
$$




$$
\begin{aligned}
\leq & {\left[\mathbf{L}^{R R}\left(\left|\mathbf{A}^{R}\right|+\left|\mathbf{B}^{R}\right|+\left|\mathbf{Q}^{R}\right|\right)+\mathbf{L}^{I R}\left(\left|\mathbf{A}^{I}\right|+\left|\mathbf{B}^{I}\right|+\left|\mathbf{Q}^{I}\right|\right)\right]\left\|\tilde{\mathbf{u}}^{R}-\tilde{\mathbf{u}}^{\prime R}\right\|_{L^{2}}^{2} } \\
& +\left[\mathbf{L}^{R I}\left(\left|\mathbf{A}^{R}\right|+\left|\mathbf{B}^{R}\right|+\left|\mathbf{Q}^{R}\right|\right)+\mathbf{L}^{I I}\left(\left|\mathbf{A}^{I}\right|+\left|\mathbf{B}^{I}\right|+\left|\mathbf{Q}^{I}\right|\right)\right]\left\|\tilde{\mathbf{u}}^{I}-\tilde{\mathbf{u}}^{\prime I}\right\|_{L^{2}}^{2}, \\
\mathbf{T}\left\|\tilde{\mathbf{u}}^{I}-\tilde{\mathbf{u}}^{I}\right\|_{L^{2}}^{2} & \\
\leq & {\left[\mathbf{L}^{I R}\left(\left|\mathbf{A}^{R}\right|+\left|\mathbf{B}^{R}\right|+\left|\mathbf{Q}^{R}\right|\right)+\mathbf{L}^{R R}\left(\left|\mathbf{A}^{I}\right|+\left|\mathbf{B}^{I}\right|+\left|\mathbf{Q}^{I}\right|\right)\right]\left\|\tilde{\mathbf{u}}^{R}-\tilde{\mathbf{u}}^{\prime R}\right\|_{L^{2}}^{2} } \\
& +\left[\mathbf{L}^{I I}\left(\left|\mathbf{A}^{R}\right|+\left|\mathbf{B}^{R}\right|+\left|\mathbf{Q}^{R}\right|\right)+\mathbf{L}^{R I}\left(\left|\mathbf{A}^{I}\right|+\left|\mathbf{B}^{I}\right|+\left|\mathbf{Q}^{I}\right|\right)\right]\left\|\tilde{\mathbf{u}}^{I}-\tilde{\mathbf{u}}^{I}\right\|_{L^{2}}^{2} .
\end{aligned}
$$

The inequalities (13) and (14) can be rewritten as $(\tilde{\mathbf{T}}-\tilde{\mathbf{P}} \tilde{\mathbf{L}})\left\|\tilde{\boldsymbol{\alpha}}-\tilde{\boldsymbol{\alpha}}^{\prime}\right\|_{L^{2}}^{2} \leq \mathbf{0}$. Because $\tilde{\mathbf{T}}-\tilde{\mathbf{P}} \tilde{\mathbf{L}}$ is a nonsingular M-matrix, it follows from Lemma 1 that the inverse matrix $(\tilde{\mathbf{T}}-\tilde{\mathbf{P}} \tilde{\mathbf{L}})^{-1}$ exists and $\operatorname{det}(\tilde{\mathbf{T}}-\tilde{\mathbf{P}} \tilde{\mathbf{L}})>0$ holds. Multiplying by $(\tilde{\mathbf{T}}-\tilde{\mathbf{P}} \tilde{\mathbf{L}})^{-1}$ on both left sides of $(\tilde{\mathbf{T}}-\tilde{\mathbf{P}} \tilde{\mathbf{L}}) \| \tilde{\boldsymbol{\alpha}}-$ $\tilde{\boldsymbol{\alpha}}^{\prime} \|_{L^{2}}^{2} \leq \mathbf{0}$, we obtain $\mathbf{E}\left\|\tilde{\boldsymbol{\alpha}}-\tilde{\boldsymbol{\alpha}}^{\prime}\right\|_{L^{2}}^{2}=\left\|\tilde{\boldsymbol{\alpha}}-\tilde{\boldsymbol{\alpha}}^{\prime}\right\|_{L^{2}}^{2} \leq \mathbf{0}$, where $\mathbf{E}$ is unit matrix with the same dimension with $\tilde{\mathbf{T}}-\tilde{\mathbf{P}} \tilde{\mathbf{L}}$. Therefore, we can conclude that $\left\|\tilde{\boldsymbol{\alpha}}-\tilde{\boldsymbol{\alpha}}^{\prime}\right\|_{L^{2}}^{2}=\mathbf{0}$, i.e. $\tilde{\boldsymbol{\alpha}}=\tilde{\boldsymbol{\alpha}^{\prime}}$, which is a contradiction with the assumption $\tilde{\boldsymbol{\alpha}} \neq \tilde{\boldsymbol{\alpha}^{\prime}}$.

To sum up, if $\boldsymbol{\alpha}, \boldsymbol{\alpha}^{\prime} \in \mathbb{R}^{2 n}$ with $\tilde{\boldsymbol{\alpha}} \neq \tilde{\boldsymbol{\alpha}}^{\prime}$, then $\mathbf{H}(\tilde{\boldsymbol{\alpha}}) \neq \mathbf{H}\left(\tilde{\boldsymbol{\alpha}}^{\prime}\right)$, which means that the map $\mathbf{H}(\tilde{\boldsymbol{\alpha}})$ is injective on $\mathbb{R}^{2 n}$.

(ii) In this part, we will prove that $\lim _{\|\tilde{\boldsymbol{\alpha}}\|_{L^{2}} \rightarrow \infty}\|\mathbf{H}(\tilde{\boldsymbol{\alpha}})\|_{L^{2}} \rightarrow \infty$.

Because $\tilde{\mathbf{T}}-\tilde{\mathbf{P}} \tilde{\mathbf{L}}$ is an M-matrix, according to Lemma 1, there exists a positive vector $\boldsymbol{\Lambda}=\left(\varsigma_{1}, \varsigma_{2}, \ldots, \varsigma_{2 n}\right)$ such that $(\tilde{\mathbf{T}}-\tilde{\mathbf{P}} \tilde{\mathbf{L}}) \boldsymbol{\Lambda}>0$. Obviously, there is a number $\delta>0$ such that $(\tilde{\mathbf{T}}-\tilde{\mathbf{P}} \tilde{\mathbf{L}}) \boldsymbol{\Lambda}>\delta \mathbf{I}$, where $\mathbf{I} \in \mathbb{R}^{2 n}$ is an unit vector.

According to (7), we define $\tilde{\mathbf{H}}(\tilde{\boldsymbol{\alpha}})=\mathbf{H}(\tilde{\boldsymbol{\alpha}})-\mathbf{H}(\mathbf{0})$, that is

$$
\tilde{\mathbf{H}}(\tilde{\boldsymbol{\alpha}})=\tilde{\boldsymbol{\pi}} \nabla(\nabla \tilde{\boldsymbol{\alpha}}) \mathbf{I}^{\prime}-\tilde{\mathbf{D}} \tilde{\boldsymbol{\alpha}}+\mathbf{P}_{1}\left[\tilde{\mathbf{G}}^{R}(\tilde{\boldsymbol{\alpha}})-\tilde{\mathbf{G}}^{R}(\mathbf{0})\right]+\mathbf{P}_{2}\left[\tilde{\mathbf{G}}^{I}(\tilde{\boldsymbol{\alpha}})-\tilde{\mathbf{G}}^{I}(\mathbf{0})\right]
$$

It is obvious that, if $\lim _{\|\tilde{\boldsymbol{\alpha}}\|_{L^{2}}^{2} \rightarrow \infty}\|\tilde{\mathbf{H}}(\tilde{\boldsymbol{\alpha}})\|_{L^{2}}^{2} \rightarrow \infty$, then $\lim _{\|\tilde{\boldsymbol{\alpha}}\|_{L^{2}}^{2} \rightarrow \infty}\|\mathbf{H}(\tilde{\boldsymbol{\alpha}})\|_{L^{2}}^{2} \rightarrow \infty$.

Multiplying by $\tilde{\boldsymbol{\alpha}}^{T}$ on both sides of (15), we obtain

$$
\tilde{\boldsymbol{\alpha}}^{\mathrm{T}} \tilde{\mathbf{H}}(\tilde{\boldsymbol{\alpha}})=\tilde{\boldsymbol{\alpha}}^{\mathrm{T}} \tilde{\boldsymbol{\pi}} \nabla(\nabla \tilde{\boldsymbol{\alpha}}) \mathbf{I}^{\prime}-\tilde{\boldsymbol{\alpha}}^{\mathrm{T}} \tilde{\mathbf{D}} \tilde{\boldsymbol{\alpha}}+\tilde{\boldsymbol{\alpha}}^{\mathrm{T}} \mathbf{P}_{1}\left[\tilde{\mathbf{G}}^{R}(\tilde{\boldsymbol{\alpha}})-\tilde{\mathbf{G}}^{R}(\mathbf{0})\right]+\tilde{\boldsymbol{\alpha}}^{\mathrm{T}} \mathbf{P}_{2}\left[\tilde{\mathbf{G}}^{I}(\tilde{\boldsymbol{\alpha}})-\tilde{\mathbf{G}}^{I}(\mathbf{0})\right]
$$

Integrating on domain $\Omega$ on both sides of (16), we obtain

$$
\begin{aligned}
& \int_{\Omega} \tilde{\boldsymbol{\alpha}}^{\mathrm{T}} \tilde{\mathbf{H}}(\tilde{\boldsymbol{\alpha}}) \mathrm{d} \mathbf{z} \\
& =\int_{\Omega} \tilde{\boldsymbol{\alpha}}^{\mathrm{T}} \tilde{\boldsymbol{\pi}} \nabla(\nabla \tilde{\boldsymbol{\alpha}}) \mathbf{I}^{\prime} \mathrm{d} \mathbf{z}-\int_{\Omega} \tilde{\mathbf{D}} \tilde{\boldsymbol{\alpha}}^{\mathrm{T}} \tilde{\boldsymbol{\alpha}} \mathrm{d} \mathbf{z}+\int_{\Omega} \mathbf{P}_{1} \tilde{\boldsymbol{\alpha}}^{\mathrm{T}}\left[\tilde{\mathbf{G}}^{R}(\tilde{\boldsymbol{\alpha}})-\tilde{\mathbf{G}}^{R}(\mathbf{0})\right] \mathrm{d} \mathbf{z} \\
& \quad+\int_{\Omega} \mathbf{P}_{2} \tilde{\boldsymbol{\alpha}}^{\mathrm{T}}\left[\tilde{\mathbf{G}}^{I}(\tilde{\boldsymbol{\alpha}})-\tilde{\mathbf{G}}^{I}(\mathbf{0})\right] \mathrm{d} \mathbf{z} .
\end{aligned}
$$

By virtue of inequalities (13) and (14), we get

$$
\begin{aligned}
\int_{\Omega} \tilde{\boldsymbol{\alpha}}^{\mathrm{T}} \tilde{\mathbf{H}}(\tilde{\boldsymbol{\alpha}}) \mathrm{d} \mathbf{z} & \\
\leq & -\left\|\tilde{\boldsymbol{\alpha}}^{T}\right\|_{L^{2}}\left[\begin{array}{cc}
\mathbf{T} & 0 \\
0 & \mathbf{T}
\end{array}\right]\|\tilde{\boldsymbol{\alpha}}\|_{L^{2}} \\
& +\left\|\tilde{\boldsymbol{\alpha}}^{T}\right\|_{L^{2}}\left[\begin{array}{cc}
\left|\mathbf{A}^{R}\right|+\left|\mathbf{B}^{R}\right|+\left|\mathbf{Q}^{R}\right| & \left|\mathbf{A}^{I}\right|+\left|\mathbf{B}^{I}\right|+\left|\mathbf{Q}^{I}\right| \\
\left|\mathbf{A}^{I}\right|+\left|\mathbf{B}^{I}\right|+\left|\mathbf{Q}^{I}\right| & \left|\mathbf{A}^{R}\right|+\left|\mathbf{B}^{R}\right|+\left|\mathbf{Q}^{R}\right|
\end{array}\right]\left[\begin{array}{cc}
\mathbf{L}^{R R} & \mathbf{L}^{R I} \\
\mathbf{L}^{I R} & \mathbf{L}^{I I}
\end{array}\right]\|\tilde{\boldsymbol{\alpha}}\|_{L^{2}}
\end{aligned}
$$




$$
=-\left\|\tilde{\boldsymbol{\alpha}}^{T}\right\|_{L^{2}}(\tilde{\mathbf{T}}-\tilde{\mathbf{P}} \tilde{\mathbf{L}})\|\tilde{\boldsymbol{\alpha}}\|_{L^{2}}
$$

i.e.

$$
\int_{\Omega} \sum_{k^{\prime}=1}^{2 n} \tilde{\alpha}_{k^{\prime}} \tilde{H}_{k^{\prime}}(\tilde{\boldsymbol{\alpha}}) \mathrm{d} \mathbf{z} \leq \int_{\Omega} \sum_{k^{\prime}=1}^{2 n}\left\{-\tilde{T}_{k^{\prime}}\left|\tilde{\alpha}_{k^{\prime}}\right|^{2}+\sum_{j^{\prime}=1}^{2 n} \tilde{p}_{k^{\prime} j^{\prime}} \tilde{l}_{k^{\prime} j^{\prime}}\left|\tilde{\alpha}_{j^{\prime}}\right|^{2}\right\} \mathrm{d} \mathbf{z} .
$$

Multiplying by $\varsigma_{k^{\prime}}$ on both sides of (17), we obtain

$$
\int_{\Omega} \sum_{k^{\prime}=1}^{2 n} \varsigma_{k^{\prime}} \tilde{\alpha}_{k^{\prime}} \tilde{H}_{k^{\prime}}(\tilde{\boldsymbol{\alpha}}) \mathrm{d} \mathbf{z} \leq \int_{\Omega} \sum_{k^{\prime}=1}^{2 n} \varsigma_{k^{\prime}}\left\{-\tilde{T}_{k^{\prime}}\left|\tilde{\alpha}_{k^{\prime}}\right|^{2}+\sum_{j^{\prime}=1}^{2 n} \tilde{p}_{k^{\prime} j^{\prime}} \tilde{l}_{k^{\prime} j^{\prime}}\left|\tilde{\alpha}_{j^{\prime}}\right|^{2}\right\} \mathrm{d} \mathbf{z} .
$$

Furthermore, the above inequality can be rewritten as $\delta\|\tilde{\boldsymbol{\alpha}}\|_{L^{2}}^{2} \leq \max _{k^{\prime} \in \Theta}\left\{\varsigma_{k^{\prime}}\right\}\|\tilde{\boldsymbol{\alpha}}\|_{L^{2}} \times$ $\|\tilde{\mathbf{H}}(\tilde{\boldsymbol{\alpha}})\|_{L^{2}}$. Namely, $\delta\|\tilde{\boldsymbol{\alpha}}\|_{L^{2}} \leq \max _{k^{\prime} \in \Theta}\left\{\zeta_{k^{\prime}}\right\}\|\tilde{\mathbf{H}}(\tilde{\boldsymbol{\alpha}})\|_{L^{2}}$. Obviously, we can obtain $\lim _{\|\tilde{\boldsymbol{\alpha}}\|_{L^{2}} \rightarrow \infty}\|\tilde{\mathbf{H}}(\tilde{\boldsymbol{\alpha}})\|_{L^{2}} \rightarrow \infty$, which is $\lim _{\|\tilde{\boldsymbol{\alpha}}\|_{L^{2}} \rightarrow \infty}\|\mathbf{H}(\tilde{\boldsymbol{\alpha}})\|_{L^{2}} \rightarrow \infty$.

Combining (i) and (ii) we can conclude from Lemma 2 that the map $\mathbf{H}(\tilde{\boldsymbol{\alpha}})$ is a homeomorphism on $\mathbb{R}^{2 n}$. Therefore, (1) has a unique equilibrium state. The proof is completed.

Next, a theorem will be proposed to guarantee the global exponential stability of the equilibrium state of (1).

For convenience, we translate the coordinates of (3) and (4). Let $u_{k}=\tilde{u}_{k}-\tilde{u}_{k}^{\#}=u_{k}^{R}+i u_{k}^{I}$, $k \in \Theta$. After this translation, we have

$$
\begin{aligned}
& \left\{\begin{aligned}
& \frac{\partial u_{k}^{R}(t, \mathbf{z})}{\partial t}= \pi_{k} \sum_{h=1}^{m} \frac{\partial}{\partial z_{h}}\left[\frac{\partial u_{k}^{R}(t, \mathbf{z})}{\partial z_{h}}\right]-d_{k} u_{k}^{R}(t, \mathbf{z}) \\
&+\sum_{j=1}^{n}\left[a_{k j}^{R} g_{j}^{R}\left(u_{j}^{R}(t, \mathbf{z}), u_{j}^{I}(t, \mathbf{z})\right)-a_{k j}^{I} g_{j}^{I}\left(u_{j}^{R}(t, \mathbf{z}), u_{j}^{I}(t, \mathbf{z})\right)\right] \\
&+\sum_{j=1}^{n}\left[b_{k j}^{R} g_{j}^{R}\left(u_{j}^{R}\left(t-\tau_{k j}(t), \mathbf{z}\right), u_{j}^{I}\left(t-\tau_{k j}(t), \mathbf{z}\right)\right)\right. \\
&\left.-b_{k j}^{I} g_{j}^{I}\left(u_{j}^{R}\left(t-\tau_{k j}(t), \mathbf{z}\right), u_{j}^{I}\left(t-\tau_{k j}(t), \mathbf{z}\right)\right)\right] \\
&+\sum_{j=1}^{n}\left[q_{k j}^{R} \int_{-\infty}^{t} \sigma_{k j}(t-s) g_{j}^{R}\left(u_{j}^{R}(s, \mathbf{z}), u_{j}^{I}(s, \mathbf{z})\right) \mathrm{d} s\right. \\
&\left.-q_{k j}^{I} \int_{-\infty}^{t} \sigma_{k j}(t-s) g_{j}^{I}\left(u_{j}^{R}(s, \mathbf{z}), u_{j}^{I}(s, \mathbf{z})\right) \mathrm{d} s\right] \\
&\left.u_{k}^{R}(t, \mathbf{z})\right|_{\mathbf{z} \in \partial \Omega}=0,
\end{aligned}\right. \\
& \left\{\begin{aligned}
& \frac{\partial u_{k}^{I}(t, \mathbf{z})}{\partial t}= \pi_{k} \sum_{h=1}^{m} \frac{\partial}{\partial z_{h}}\left[\frac{\partial u_{k}^{I}(t, \mathbf{z})}{\partial z_{h}}\right]-d_{k} u_{k}^{I}(t, \mathbf{z}) \\
&+\sum_{j=1}^{n}\left[a_{k j}^{R} g_{j}^{I}\left(u_{j}^{R}(t, \mathbf{z}), u_{j}^{I}(t, \mathbf{z})\right)+a_{k j}^{I} g_{j}^{R}\left(u_{j}^{R}(t, \mathbf{z}), u_{j}^{I}(t, \mathbf{z})\right)\right] \\
&+\sum_{j=1}^{n}\left[b_{k j}^{R} g_{j}^{I}\left(u_{j}^{R}\left(t-\tau_{k j}(t), \mathbf{z}\right), u_{j}^{I}\left(t-\tau_{k j}(t), \mathbf{z}\right)\right)\right. \\
&\left.+b_{k j}^{I} g_{j}^{R}\left(u_{j}^{R}\left(t-\tau_{k j}(t), \mathbf{z}\right), u_{j}^{I}\left(t-\tau_{k j}(t), \mathbf{z}\right)\right)\right] \\
&+\sum_{j=1}^{n}\left[q_{k j}^{R} \int_{-\infty}^{t} \sigma_{k j}(t-s) g_{j}^{I}\left(u_{j}^{R}(s, \mathbf{z}), u_{j}^{I}(s, \mathbf{z})\right) \mathrm{d} s\right. \\
&\left.+q_{k j}^{I} \int_{-\infty}^{t} \sigma_{k j}(t-s) g_{j}^{R}\left(u_{j}^{R}(s, \mathbf{z}), u_{j}^{I}(s, \mathbf{z})\right) \mathrm{d} s\right] \\
&\left.u_{k}^{I}(t, \mathbf{z})\right|_{\mathbf{z} \in \partial \Omega}=0
\end{aligned}\right.
\end{aligned}
$$

where $g_{j}^{R}\left(u_{j}^{R}, u_{j}^{I}\right)=\tilde{g}_{j}^{R}\left(\tilde{u}_{j}^{R}, \tilde{u}_{j}^{I}\right)-\tilde{g}_{j}^{R}\left(\tilde{u}_{j}^{\# R}, \tilde{u}_{j}^{\# I}\right), g_{j}^{I}\left(u_{j}^{R}, u_{j}^{I}\right)=\tilde{g}_{j}^{I}\left(\tilde{u}_{j}^{R}, \tilde{u}_{j}^{I}\right)-\tilde{g}_{j}^{I}\left(\tilde{u}_{j}^{\# R}, \tilde{u}_{j}^{\# I}\right)$,

$$
\boldsymbol{\alpha}=\left(\left(\mathbf{u}^{R}\right)^{T},\left(\mathbf{u}^{I}\right)^{\mathrm{T}}\right)^{T}, \quad \mathbf{G}^{R}(\boldsymbol{\alpha})=\left(\left(\mathbf{g}^{R}\left(\mathbf{u}^{R}, \mathbf{u}^{I}\right)\right)^{\mathrm{T}},\left(\mathbf{g}^{R}\left(\mathbf{u}^{R}, \mathbf{u}^{I}\right)\right)^{T}\right)^{\mathrm{T}},
$$




$$
\mathbf{G}^{I}(\boldsymbol{\alpha})=\left(\left(\mathbf{g}^{I}\left(\mathbf{u}^{R}, \mathbf{u}^{I}\right)\right)^{\mathrm{T}},\left(\mathbf{g}^{I}\left(\mathbf{u}^{R}, \mathbf{u}^{I}\right)\right)^{T}\right)^{\mathrm{T}}
$$

The initial conditions of (18) and (19) are $\varphi_{k}(s, \mathbf{z})=\tilde{\varphi}_{k}(s, \mathbf{z})-\tilde{u}_{k}^{\#}, k \in \Theta$.

Clearly, the exponential stability of zero solutions of the system being composed of (18) and (19) is equivalent to the exponential stability of the equilibrium state of (1). Next, we propose a theorem to ensure the global exponential stability of the equilibrium state of (18) and (19).

Theorem 2 The equilibrium state $\tilde{\mathbf{u}}^{\#}$ of (1) is globally exponentially stable for all $\mathbf{J} \in \mathbb{C}^{n}$ and $\mathbf{z} \in \Omega$ if all conditions of the Theorem 1 hold.

Proof If the conditions of Theorem 2 hold, then (1) has a unique equilibrium state according to Theorem 1. That is to say, the RDCVNNs composed of (18) and (19) has a unique zero solution.

The matrix $\tilde{\mathbf{T}}-\tilde{\mathbf{P}} \tilde{\mathbf{L}}$ is an M-matrix, so from Lemma 1, there exists a positive vector $\Lambda=$ $\left(\varsigma_{1}, \varsigma_{2}, \ldots, \varsigma_{2 n}\right)$ such that the inequalities (20) and (21) hold, where, when $k^{\prime} \in \Theta, \varsigma_{k^{\prime}}=\beta_{k}$ and $k=k^{\prime}$; and when $k^{\prime} \in \widehat{\Theta}-\Theta, \varsigma_{k^{\prime}}=\gamma_{k}$ and $k=k^{\prime}-n$,

$$
\begin{aligned}
& \left\{\left(-2 \frac{m \pi_{k}}{\hat{\omega}^{2}}-2 d_{k}\right)+\sum_{j=1}^{n}\left[\left(l_{j}^{R R}+l_{j}^{R I}\right)\left(\left|a_{k j}^{R}\right|+\left|b_{k j}^{R}\right|+\left|q_{k j}^{R}\right|\right)\right.\right. \\
& \left.\left.\quad+\left(l_{j}^{I R}+l_{j}^{I I}\right)\left(\left|a_{k j}^{I}\right|+\left|b_{k j}^{I}\right|+\left|q_{k j}^{I}\right|\right)\right]\right\} \beta_{k} \\
& \quad+\sum_{j=1}^{n} \beta_{j}\left[\left(\left|a_{k j}^{R}\right| l_{j}^{R R}+\left|a_{k j}^{I}\right| l_{j}^{I R}\right)+\left(\left|b_{k j}^{R}\right| l_{j}^{R R}+\left|b_{k j}^{I}\right| l_{j}^{I R}\right)+\left(\left|q_{k j}^{R}\right| l_{j}^{R R}+\left|q_{k j}^{I}\right| l_{j}^{I R}\right)\right] \\
& \quad+\sum_{j=1}^{n} \gamma_{j}\left[\left(\left|a_{k j}^{R}\right| l_{k}^{R I}+\left|a_{k j}^{I}\right| l_{j}^{I I}\right)+\left(\left|b_{k j}^{R}\right| l_{j}^{R I}+\left|b_{k j}^{I}\right| l_{j}^{I I}\right)+\left(\left|q_{k j}^{R}\right| l_{j}^{R I}+\left|q_{k j}^{I}\right| l_{j}^{I I}\right)\right]<0, \\
& \quad+2 \frac{m \pi_{k}}{\hat{\omega}^{2}}-2 d_{k}+\sum_{j=1}^{n}\left[\left(l_{j}^{I R}+l_{j}^{I I}\right)\left(\left|a_{k j}^{R}\right|+\left|b_{k j}^{R}\right|+\left|q_{k j}^{R}\right|\right)\right. \\
& \left.\left.\quad+\left(l_{j}^{R R}+l_{j}^{R I}\right)\left(\left|a_{k j}^{I}\right|+\left|b_{k j}^{I}\right|+\left|q_{k j}^{I}\right|\right)\right]\right\} \gamma_{k} \\
& \quad+\sum_{j=1}^{n} \beta_{j}\left[\left(\left|a_{k j}^{R}\right| l_{j}^{I R}+\left|a_{k j}^{I}\right| l_{j}^{R R}\right)+\left(\left|b_{k j}^{R}\right| l_{j}^{I R}+\left|b_{k j}^{I}\right| l_{j}^{R R}\right)+\left(\left|q_{k j}^{R}\right| l_{j}^{I R}+\left|q_{k j}^{I}\right| l l_{j}^{R R}\right)\right] \\
& \quad+\sum_{j=1}^{n} \gamma_{j}\left[\left(\left|a_{k j}^{R}\right| l l_{k}^{I I}+\left|a_{k j}^{I}\right| l_{j}^{R I}\right)+\left(\left|b_{k j}^{R}\right| l_{j}^{I I}+\left|b_{k j}^{I}\right| l_{j}^{R I}\right)+\left(\left|q_{k j}^{R}\right| l l_{j}^{I I}+\left|q_{k j}^{I}\right| l_{j}^{R I}\right)\right]<0 .
\end{aligned}
$$

We construct functions by means of (20) and (21) as follows:

$$
\begin{aligned}
& F_{k}^{R}(\varepsilon) \\
& \quad=\left\{\varepsilon-2 \frac{m \pi_{k}}{\hat{\omega}^{2}}-2 d_{k}+\sum_{j=1}^{n}\left[\left(l_{j}^{R R}+l_{j}^{R I}\right)\left(\left|a_{k j}^{R}\right|+\left|b_{k j}^{R}\right|+\left|q_{k j}^{R}\right|\right)\right.\right.
\end{aligned}
$$




$$
\begin{aligned}
& \left.\left.+\left(l_{j}^{I R}+l_{j}^{I I}\right)\left(\left|a_{k j}^{I}\right|+\left|b_{k j}^{I}\right|+\left|q_{k j}^{I}\right|\right)\right]\right\} \beta_{k} \\
& +\sum_{j=1}^{n}\left[\left(\left|a_{k j}^{R}\right| l_{j}^{R R}+\left|a_{k j}^{I}\right| l_{j}^{I R}\right)+\left(\left|b_{k j}^{R}\right| l_{j}^{R R}+\left|b_{k j}^{I}\right| l_{j}^{I R}\right) e^{\varepsilon \tau}+\left(\left|q_{k j}^{R}\right| l_{j}^{R R}+\left|q_{k j}^{I}\right| l_{j}^{I R}\right) \mu_{k j}(\varepsilon)\right] \beta_{j} \\
& +\sum_{j=1}^{n}\left[\left(\left|a_{k j}^{R}\right| l_{k}^{R I}+\left|a_{k j}^{I}\right| l_{j}^{I I}\right)+\left(\left|b_{k j}^{R}\right| l_{j}^{R I}+\left|b_{k j}^{I}\right| l_{j}^{I I}\right) e^{\varepsilon \tau}\right. \\
& \left.+\left(\left|q_{k j}^{R}\right| l_{j}^{R I}+\left|q_{k j}^{I}\right| l_{j}^{I I}\right) \mu_{k j}(\varepsilon)\right] \gamma_{j},
\end{aligned}
$$

$F_{k}^{I}(\varepsilon)$

$$
\begin{aligned}
= & \left\{\varepsilon-2 \frac{m \pi_{k}}{\hat{\omega}^{2}}-2 d_{k}+\sum_{j=1}^{n}\left[\left(l_{j}^{I R}+l_{j}^{I I}\right)\left(\left|a_{k j}^{R}\right|+\left|b_{k j}^{R}\right|+\left|q_{k j}^{R}\right|\right)\right.\right. \\
& \left.\left.+\left(l_{j}^{R R}+l_{j}^{R I}\right)\left(\left|a_{k j}^{I}\right|+\left|b_{k j}^{I}\right|+\left|q_{k j}^{I}\right|\right)\right]\right\} \gamma_{k} \\
& +\sum_{j=1}^{n}\left[\left(\left|a_{k j}^{R}\right| l_{j}^{I R}+\left|a_{k j}^{I}\right| l_{j}^{R R}\right)+\left(\left|b_{k j}^{R}\right| l_{j}^{I R}+\left|b_{k j}^{I}\right| l_{j}^{R R}\right) e^{\varepsilon \tau}+\left(\left|q_{k j}^{R}\right| l_{j}^{I R}+\left|q_{k j}^{I}\right| l_{j}^{R R}\right) \mu_{k j}(\varepsilon)\right] \beta_{j} \\
& +\sum_{j=1}^{n}\left[\left(\left|a_{k j}^{R}\right| l_{k}^{I I}+\left|a_{k j}^{I}\right| l_{j}^{R I}\right)+\left(\left|b_{k j}^{R}\right| l_{j}^{I I}+\left|b_{k j}^{I}\right| l_{j}^{R I}\right) e^{\varepsilon \tau}\right. \\
& \left.+\left(\left|q_{k j}^{R}\right| l_{j}^{I I}+\left|q_{k j}^{I}\right| l_{j}^{R I}\right) \mu_{k j}(\varepsilon)\right] \gamma_{j} .
\end{aligned}
$$

Because $F_{k}^{R}(\varepsilon)$ and $F_{k}^{I}(\varepsilon)$ are continuous with respect to $\varepsilon, F_{k}^{R}(0)<0$ and $F_{k}^{I}(0)<0$ hold according to (20) and (21). Therefore, there exists a constant $\lambda>0$ such that $F_{k}^{R}(\lambda)<0$ and $F_{k}^{I}(\lambda)<0$ hold.

The candidate vector Lyapunov functions are chosen as follows:

$$
V_{k^{\prime}}\left(t, \alpha_{k^{\prime}}(t, \mathbf{z})\right)=\frac{1}{2} e^{\lambda t}\left\|\alpha_{k^{\prime}}(t, \mathbf{z})\right\|_{L^{2}}^{2}=\frac{1}{2} e^{\lambda t} \int_{\Omega}\left|\alpha_{k^{\prime}}(t, \mathbf{z})\right|^{2} \mathrm{~d} \mathbf{z}, \quad k^{\prime} \in \widehat{\Theta},
$$

i.e.

$$
\begin{aligned}
& V_{k^{\prime}}\left(t, \alpha_{k^{\prime}}(t, \mathbf{z})\right) \\
& \quad= \begin{cases}\frac{1}{2} e^{\lambda t}\left\|\alpha_{k}(t, \mathbf{z})\right\|_{L^{2}}^{2}=\frac{1}{2} e^{\lambda t} \int_{\Omega}\left(u_{k}^{R}(t, \mathbf{z})\right)^{2} \mathrm{~d} \mathbf{z}, & k^{\prime} \in \Theta, k=k^{\prime} \\
\frac{1}{2} e^{\lambda t}\left\|\alpha_{k^{\prime}}(t, \mathbf{z})\right\|_{L^{2}}^{2}=\frac{1}{2} e^{\lambda t} \int_{\Omega}\left(u_{k}^{I}(t, \mathbf{z})\right)^{2} \mathrm{~d} \mathbf{z}, & k^{\prime} \in \widehat{\Theta}-\Theta, k=k^{\prime}-n .\end{cases}
\end{aligned}
$$

In order to simplify the expression, let $V_{k^{\prime}}(t, \mathbf{z})$ denote $V_{k^{\prime}}\left(t, \alpha_{k^{\prime}}(t, \mathbf{z})\right)$ if there is no confusion, here $k^{\prime} \in \widehat{\Theta}$.

(1) When $k^{\prime} \in \Theta$, calculating the upper right derivative $\mathrm{D}^{+} V_{k^{\prime}}\left(t, \alpha_{k^{\prime}}(t)\right)$ along (18), we obtain

$$
\begin{aligned}
& \mathrm{D}^{+} V_{k^{\prime}}(t, \mathbf{z}) \\
& \quad=0.5 \lambda e^{\lambda t} \int_{\Omega}\left(u_{k}^{R}(t, \mathbf{z})\right)^{2} \mathrm{~d} \mathbf{z}+e^{\lambda t} \pi_{k} \sum_{h=1}^{m} \int_{\Omega} u_{k}^{R}(t, \mathbf{z}) \frac{\partial}{\partial z_{h}}\left[\frac{\partial u_{k}^{R}(t, \mathbf{z})}{\partial z_{h}}\right] \mathrm{d} \mathbf{z}
\end{aligned}
$$


Xu et al. Advances in Difference Equations

(2021) 2021:58

Page 14 of 27

$$
\begin{aligned}
& +e^{\lambda t} \sum_{h=1}^{m} \int_{\Omega} u_{k}^{R}(t, \mathbf{z})\left\{-d_{k} u_{k}^{R}(t, \mathbf{z})+\sum_{j=1}^{n}\left[a_{k j}^{R} g_{j}^{R}\left(u_{j}^{R}(t, \mathbf{z}), u_{j}^{I}(t, \mathbf{z})\right)\right.\right. \\
& \left.-a_{k j}^{I} g_{j}^{I}\left(u_{j}^{R}(t, \mathbf{z}), u_{j}^{I}(t, \mathbf{z})\right)\right] \\
& +\sum_{j=1}^{n}\left[b_{k j}^{R} g_{j}^{R}\left(u_{j}^{R}\left(t-\tau_{k j}(t), \mathbf{z}\right), u_{j}^{I}\left(t-\tau_{k j}(t), \mathbf{z}\right)\right)\right. \\
& \left.-b_{k j}^{I} g_{j}^{I}\left(u_{j}^{R}\left(t-\tau_{k j}(t), \mathbf{z}\right), u_{j}^{I}\left(t-\tau_{k j}(t), \mathbf{z}\right)\right)\right] \\
& +\sum_{j=1}^{n}\left[q_{k j}^{R} \int_{-\infty}^{t} \sigma_{k j}(t-s) g_{j}^{R}\left(u_{j}^{R}(s, \mathbf{z}), u_{j}^{I}(s, \mathbf{z})\right) \mathrm{d} s\right. \\
& \left.\left.-q_{k j}^{I} \int_{-\infty}^{t} \sigma_{k j}(t-s) g_{j}^{I}\left(u_{j}^{R}(s, \mathbf{z}), u_{j}^{I}(s, \mathbf{z})\right) \mathrm{d} s\right]\right\} \mathrm{d} \mathbf{z} .
\end{aligned}
$$

According to integration by parts, and considering Lemma 3 and Assumptions 1-4, we have

$$
\begin{aligned}
\mathrm{D}^{+} & V_{k^{\prime}}(t, \mathbf{z}) \\
= & 0.5 \lambda e^{\lambda t} \int_{\Omega}\left(u_{k}^{R}(t, \mathbf{z})\right)^{2} \mathrm{~d} \mathbf{z}-e^{\lambda t} \pi_{k} \sum_{h=1}^{m} \int_{\Omega} \frac{\partial u_{k}^{R}(t, \mathbf{z})}{\partial z_{h}} \mathrm{~d} \mathbf{z} \\
& +e^{\lambda t} \int_{\Omega} u_{k}^{R}(t, \mathbf{z})\left\{-d_{k} u_{k}^{R}(t, \mathbf{z})+\sum_{j=1}^{n}\left[a_{k j}^{R} g_{j}^{R}\left(u_{j}^{R}(t, \mathbf{z}), u_{j}^{I}(t, \mathbf{z})\right)\right.\right. \\
& \left.-a_{k j}^{I} g_{j}^{I}\left(u_{j}^{R}(t, \mathbf{z}), u_{j}^{I}(t, \mathbf{z})\right)\right] \\
& +\sum_{j=1}^{n}\left[b_{k j}^{R} g_{j}^{R}\left(u_{j}^{R}\left(t-\tau_{k j}(t), \mathbf{z}\right), u_{j}^{I}\left(t-\tau_{k j}(t), \mathbf{z}\right)\right)\right. \\
& \left.-b_{k j}^{I} g_{j}^{I}\left(u_{j}^{R}\left(t-\tau_{k j}(t), \mathbf{z}\right), u_{j}^{I}\left(t-\tau_{k j}(t), \mathbf{z}\right)\right)\right] \\
& +\sum_{j=1}^{n}\left[q_{k j}^{R} \int_{-\infty}^{t} \sigma_{k j}(t-s) g_{j}^{R}\left(u_{j}^{R}(s, \mathbf{z}), u_{j}^{I}(s, \mathbf{z})\right) \mathrm{d} s\right. \\
& \left.\left.-q_{k j}^{I} \int_{-\infty}^{t} \sigma_{k j}(t-s) g_{j}^{I}\left(u_{j}^{R}(s, \mathbf{z}), u_{j}^{I}(s, \mathbf{z})\right) \mathrm{d} s\right]\right\} \mathrm{d} \mathbf{z} \\
\leq & 0.5 \lambda e^{\lambda t} \int_{\Omega}\left(u_{k}^{R}(t, \mathbf{z})\right)^{2} \mathrm{~d} \mathbf{z}-e^{\lambda t} \int_{\Omega}\left(\frac{m \pi_{k}}{\hat{\omega}^{2}}+d_{k}\right)\left(u_{k}^{R}(t, z)\right)^{2} \mathrm{~d} \mathbf{z} \\
& +e^{\lambda t} \int_{\Omega}\left\{| u _ { k } ^ { R } ( t , \mathbf { z } ) | \sum _ { j = 1 } ^ { n } \left[\left|a_{k j}^{R}\right|\left[l_{j}^{R R}\left|u_{j}^{R}(t, \mathbf{z})\right|+l_{j}^{R I}\left|u_{j}^{I}(t, \mathbf{z})\right|\right]\right.\right. \\
& \left.+\left|a_{k j}^{I}\right|\left[l_{j}^{I R}\left|u_{j}^{R}(t, \mathbf{z})\right|+l_{j}^{I I}\left|u_{j}^{I}(t, \mathbf{z})\right|\right]\right] \\
& +\sum_{j=1}^{n}\left[\left|b_{k j}^{R}\right| \cdot\left[l_{j}^{R R}\left|u_{j}^{R}\left(t-\tau_{k j}(t), \mathbf{z}\right)\right|+l_{j}^{R I} \mid u_{j}^{I}\left(t-\tau_{k j}(t), \mathbf{z}\right)\right) \mid\right] \\
& \left.\left.\left|b_{k j}^{I}\right|\left[l_{j}^{I R}\left|u_{j}^{R}\left(t-\tau_{k j}(t), \mathbf{z}\right)\right|+l_{j}^{I I} \mid u_{j}^{I}\left(t-\tau_{k j}(t), \mathbf{z}\right)\right) \mid\right]\right] \\
&
\end{aligned}
$$


Xu et al. Advances in Difference Equations

(2021) 2021:58

Page 15 of 27

$$
\begin{aligned}
& +\sum_{j=1}^{n}\left[\left|q_{k j}^{R}\right| \int_{-\infty}^{t} \sigma_{k j}(t-s)\left[l_{j}^{R R}\left|u_{j}^{R}(s, \mathbf{z})\right|+l_{j}^{R I}\left|u_{j}^{I}(s, \mathbf{z})\right|\right] \mathrm{d} s\right. \\
& \left.\left.+\left|q_{k j}^{I}\right| \int_{-\infty}^{t} \sigma_{k j}(t-s)\left[l_{j}^{I R}\left|u_{j}^{R}(s, \mathbf{z})\right|+l_{j}^{I I}\left|u_{j}^{I}(s, \mathbf{z})\right|\right] \mathrm{d} s\right]\right\} \mathrm{d} \mathbf{z} .
\end{aligned}
$$

By using the Holder inequality, we get

$$
\begin{aligned}
& \mathrm{D}^{+} V_{k^{\prime}}\left(t, \alpha_{k^{\prime}}(t, \mathbf{z})\right) \\
& \leq 0.5 \lambda e^{\lambda t} \int_{\Omega}\left(u_{k}^{R}(t, \mathbf{z})\right)^{2} \mathrm{~d} \mathbf{z}-e^{\lambda t} \int_{\Omega}\left(\frac{m \pi_{k}}{\hat{\omega}^{2}}+d_{k}\right)\left(u_{k}^{R}(t, \mathbf{z})\right)^{2} \mathrm{~d} \mathbf{z} \\
& +e^{\lambda t} \int_{\Omega}\left\{0 . 5 \sum _ { j = 1 } ^ { n } \left\{| a _ { k j } ^ { R } | \left\{l_{j}^{R R}\left[\left(u_{k}^{R}(t, \mathbf{z})\right)^{2}+\left(u_{j}^{R}(t, \mathbf{z})\right)^{2}\right]\right.\right.\right. \\
& \left.+l_{j}^{R I}\left[\left(u_{k}^{R}(t, \mathbf{z})\right)^{2}+\left(u_{j}^{I}(t, \mathbf{z})\right)^{2}\right]\right\}+\left|a_{k j}^{I}\right|\left\{l_{j}^{I R}\left[\left(u_{k}^{R}(t, \mathbf{z})\right)^{2}+\left(u_{j}^{R}(t, \mathbf{z})\right)^{2}\right]\right. \\
& \left.\left.+l_{j}^{I I}\left[\left(u_{k}^{R}(t, \mathbf{z})\right)^{2}+\left(u_{j}^{I}(t, \mathbf{z})\right)^{2}\right]\right\}\right\} \\
& +0.5 \sum_{j=1}^{n}\left\{| b _ { k j } ^ { R } | \cdot \left\{l_{j}^{R R}\left[\left(u_{k}^{R}(t, \mathbf{z})\right)^{2}+\left(u_{j}^{R}\left(t-\tau_{k j}(t), \mathbf{z}\right)\right)^{2}\right]\right.\right. \\
& \left.+l_{j}^{R I}\left[\left(u_{k}^{R}(t, \mathbf{z})\right)^{2}+\left(u_{j}^{I}\left(t-\tau_{k j}(t), \mathbf{z}\right)\right)^{2}\right]\right\} \\
& +\left|b_{k j}^{I}\right|\left\{l_{j}^{I R}\left[\left(u_{k}^{R}(t, \mathbf{z})\right)^{2}+\left(u_{j}^{R}\left(t-\tau_{k j}(t), \mathbf{z}\right)\right)^{2}\right]\right. \\
& \left.\left.+l_{j}^{I I}\left[\left(u_{k}^{R}(t, \mathbf{z})\right)^{2}+\left(u_{j}^{I}\left(t-\tau_{k j}(t), \mathbf{z}\right)\right)^{2}\right]\right\}\right\} \\
& +0.5 \sum_{j=1}^{n}\left\{| q _ { k j } ^ { R } | \int _ { - \infty } ^ { t } \sigma _ { k j } ( t - s ) \left\{l_{j}^{R R}\left[\left(u_{k}^{R}(t, \mathbf{z})\right)^{2}+\left(u_{j}^{R}(s, \mathbf{z})\right)^{2}\right]\right.\right. \\
& \left.+l_{j}^{R I}\left[\left(u_{k}^{R}(t, \mathbf{z})\right)^{2}+\left(u_{j}^{I}(s, \mathbf{z})\right)^{2}\right]\right\} \mathrm{d} s \\
& +\left\{| q _ { k j } ^ { I } | \int _ { - \infty } ^ { t } \sigma _ { k j } ( t - s ) \left\{l l_{j}^{I R}\left[\left(u_{k}^{R}(t, \mathbf{z})\right)^{2}+\left(u_{j}^{R}(s, \mathbf{z})\right)^{2}\right]\right.\right. \\
& \left.\left.\left.+l_{j}^{I I}\left[\left(u_{k}^{R}(t, \mathbf{z})\right)^{2}+\left(u_{j}^{I}(s, \mathbf{z})\right)^{2}\right]\right\} \mathrm{d} s\right\}\right\} \mathrm{d} \mathbf{z} \\
& \leq 0.5 e^{\lambda t}\left\{\left\{\lambda-2 \frac{m \pi_{k}}{\hat{\omega}^{2}}-2 d_{k}+\sum_{j=1}^{n}\left(l_{j}^{R R}+l_{j}^{R I}\right)\left(\left|a_{k j}^{R}\right|+\left|b_{k j}^{R}\right|+\left|q_{k j}^{R}\right|\right)\right.\right. \\
& \left.+\left(l_{j}^{I R}+l_{j}^{I I}\right)\left(\left|a_{k j}^{I}\right|+\left|b_{k j}^{I}\right|+\left|q_{k j}^{I}\right|\right)\right\} \\
& \times \int_{\Omega}\left(u_{k}^{R}(t, \mathbf{z})\right)^{2} \mathrm{~d} \mathbf{z}+\sum_{j=1}^{n}\left[\left(\left|a_{k j}^{R}\right| l_{j}^{R R}+\left|a_{k j}^{I}\right| l_{j}^{I R}\right) \int_{\Omega}\left(u_{j}^{R}(t, \mathbf{z})\right)^{2} \mathrm{~d} \mathbf{z}\right. \\
& \left.+\left(\left|a_{k j}^{R}\right| l_{j}^{R I}+\left|a_{k j}^{I}\right| l_{j}^{I I}\right) \int_{\Omega}\left(u_{j}^{I}(t, \mathbf{z})\right)^{2} \mathrm{~d} \mathbf{z}\right] \\
& +\sum_{j=1}^{n}\left[\left(\left|b_{k j}^{R}\right| l_{j}^{R R}+\left|b_{k j}^{I}\right| l_{j}^{I R}\right) \int_{\Omega}\left(u_{j}^{R}\left(t-\tau_{k j}(t), \mathbf{z}\right)\right)^{2} \mathrm{~d} \mathbf{z}\right.
\end{aligned}
$$




$$
\begin{aligned}
& \left.+\left(\left|b_{k j}^{R}\right| l_{j}^{R I}+\left|b_{k j}^{I}\right| l_{j}^{I I}\right) \int_{\Omega}\left(u_{j}^{I}\left(t-\tau_{k j}(t), \mathbf{z}\right)\right)^{2} \mathrm{~d} \mathbf{z}\right] \\
& +\sum_{j=1}^{n}\left\{\int _ { - \infty } ^ { t } \sigma _ { k j } ( t - s ) \left[\left(\left|q_{k j}^{R}\right| l_{j}^{R R}+\left|q_{k j}^{I}\right| l_{j}^{R R}\right) \int_{\Omega}\left(u_{j}^{R}(s, \mathbf{z})\right)^{2} \mathrm{~d} \mathbf{z}+\left(\left|q_{k j}^{R}\right| l_{j}^{R I}+\left|q_{k j}^{I}\right| l_{j}^{I I}\right)\right.\right. \\
& \left.\left.\left.+\int_{\Omega}\left(u_{j}^{I}(s, \mathbf{z})\right)^{2} \mathrm{~d} \mathbf{z}\right] \mathrm{d} s\right\}\right\} \\
& \leq\left\{\lambda-2 \frac{m \pi_{k}}{\hat{\omega}^{2}}-2 d_{k}+\sum_{j=1}^{n}\left[\left(l_{j}^{R R}+l_{j}^{R I}\right)\left(\left|a_{k j}^{R}\right|+\left|b_{k j}^{R}\right|+\left|q_{k j}^{R}\right|\right)\right.\right. \\
& \left.\left.+\left(l_{j}^{I R}+l_{j}^{I I}\right)\left(\left|a_{k j}^{I}\right|+\left|b_{k j}^{I}\right|+\left|q_{k j}^{I}\right|\right)\right]\right\} V_{k}(t, \mathbf{z}) \\
& +\sum_{j=1}^{n}\left[\left(\left|a_{k j}^{R}\right| l_{j}^{R R}+\left|a_{k j}^{I}\right| l_{j}^{I R}\right) V_{j}(t, \mathbf{z})+\left(\left|a_{k j}^{R}\right| l_{j}^{R I}+\left|a_{k j}^{I}\right| l_{j}^{I I}\right) V_{j+n}(t, \mathbf{z})\right] \\
& +e^{\lambda \tau} \sum_{j=1}^{n}\left[\left(\left|b_{k j}^{R}\right| l l_{j}^{R R}+\left|b_{k j}^{I}\right| l_{j}^{I R}\right) V_{j}\left(t-\tau_{k j}(t), \mathbf{z}\right)\right. \\
& \left.+\left(\left|b_{k j}^{R}\right| l_{j}^{R I}+\left|b_{k j}^{I}\right| l_{j}^{I I}\right) V_{j+n}\left(t-\tau_{k j}(t), \mathbf{z}\right)\right] \\
& +\sum_{j=1}^{n} \int_{-\infty}^{t} \sigma_{k j}(t-s) e^{\lambda(t-s)}\left[\left(\left|q_{k j}^{R}\right| l_{j}^{R R}+\left|q_{k j}^{I}\right| l_{j}^{I R}\right) V_{j}(s, \mathbf{z})\right. \\
& \left.+\left(\left|q_{k j}^{R}\right| l_{j}^{R I}+\left|q_{k j}^{I}\right| l_{j}^{I I}\right) V_{j+n}(s, \mathbf{z})\right] \mathrm{d} s .
\end{aligned}
$$

The curve is defined by $\Upsilon=\left\{\eta(\chi): \eta_{k^{\prime}}=\varsigma_{k^{\prime}} \chi, \chi>0, k^{\prime} \in \widehat{\Theta}\right\}$, and the sets are defined by $\Pi(\boldsymbol{\eta})=\{\mathbf{w}: \mathbf{0} \leq \mathbf{w} \leq \boldsymbol{\eta}, \boldsymbol{\eta} \in \boldsymbol{s}\}$. Obviously, $\Upsilon(\boldsymbol{\eta}(\chi)) \supset \Upsilon\left(\boldsymbol{\eta}\left(\chi^{\prime}\right)\right)$ when $\chi>\chi^{\prime}$.

In what follows, it will be proven that there exist constants $\Gamma>0$ and $\lambda>0$ such that $\left\|\mathbf{u}^{R}(t, \mathbf{z})\right\|_{L^{2}} \leq \Gamma\|\boldsymbol{\varphi}(\mathbf{z})\|_{L^{2}} e^{-0.5 \lambda t}$, where $\|\boldsymbol{\varphi}(\mathbf{z})\|_{L^{2}}=\sup _{s \in(-\infty, 0]}\|\boldsymbol{\varphi}(s, \mathbf{z})\|_{L^{2}}, t \geq 0$. Let $\bar{v}=\max _{k \in \Theta}\left\{\beta_{k}, \gamma_{k}\right\}, \underline{v}=\min _{k \in \Theta}\left\{\beta_{k}, \gamma_{k}\right\}$, and $\chi_{0}=\theta\|\varphi(\mathbf{z})\|_{L_{2}}^{2} / \underline{v}$, where $\theta>1$ is a positive constant. Then

$$
\left\{\mathbf{V}: \mathbf{V}(s, \mathbf{z})=0.5 e^{\lambda s}\|\boldsymbol{\alpha}(s, \mathbf{z})\|_{L^{2}}^{2},-\infty<s \leq 0\right\} \subset \Upsilon\left(\eta_{0}\left(\chi_{0}\right)\right)
$$

so when $-\infty<s \leq 0$, we have

$$
V_{j}(s, \mathbf{z})=0.5 e^{\lambda s}\left\|u_{j}^{R}(s, \mathbf{z})\right\|_{L_{2}}^{2}<\beta_{j} \chi_{0}, \quad V_{j+n}(s, \mathbf{z})=0.5 e^{\lambda s}\left\|u_{j}^{I}(s, \mathbf{z})\right\|_{L_{2}}^{2}<\gamma_{j} \chi_{0}, \quad j \in \Theta .
$$

We can conclude that $V_{j}(t, \mathbf{z})<\beta_{j} \chi_{0}, V_{j+n}(t, \mathbf{z})<\gamma_{j} \chi_{0}, j \in \Theta$.

If it does not hold, there are some $\hat{k} \in \Theta$ and $t_{1}>0$ such that $V_{\hat{k}}\left(t_{1}, \mathbf{z}\right)=\beta_{\hat{k}} \chi_{0}$, $D^{+}\left(V_{\hat{k}}\left(t_{1}, \mathbf{z}\right)\right) \geq 0, V_{j}\left(t_{1}, \mathbf{z}\right) \leq \beta_{j} \chi_{0}, V_{j+n}\left(t_{1}, \mathbf{z}\right) \leq \gamma_{j} \chi_{0}, j \neq \hat{k}, j \in \Theta$. Substituting these into (25) and considering that $F_{k}^{R}(\lambda)<0$, we have

$$
\begin{aligned}
& \mathrm{D}^{+} V_{\hat{k}}\left(t_{1}, \alpha_{\hat{k}}\left(t_{1}, \mathbf{z}\right)\right) \\
& \quad \leq\left\{\lambda-2 \frac{m \pi_{\hat{k}}}{\hat{\omega}^{2}}-2 d_{\hat{k}}+\sum_{j=1}^{n}\left[\left(l_{j}^{R R}+l_{j}^{R I}\right)\left(\left|a_{\hat{k} j}^{R}\right|+\left|b_{\hat{k} j}^{R}\right|+\left|q_{\hat{k} j}^{R}\right|\right)\right.\right.
\end{aligned}
$$


Xu et al. Advances in Difference Equations

(2021) 2021:58

Page 17 of 27

$$
\begin{aligned}
& \left.\left.+\left(l_{j}^{I R}+l_{j}^{I I}\right)\left(\left|a_{\hat{k} j}^{I}\right|+\left|b_{\hat{k} j}^{I}\right|+\left|q_{\hat{k} j}^{I}\right|\right)\right]\right\} V_{\hat{k}}\left(t_{1}, \mathbf{z}\right) \\
& +\sum_{j=1}^{n}\left[\left(\left|a_{\hat{k} j}^{R}\right| l_{j}^{R R}+\left|a_{\hat{k} j}^{I}\right| l_{j}^{I R}\right) V_{j}\left(t_{1}, \mathbf{z}\right)+\left(\left|a_{\hat{k} j}^{R}\right| l_{j}^{R I}+\left|a_{\hat{k} j}^{I}\right| l_{j}^{I I}\right) V_{j+n}\left(t_{1}, \mathbf{z}\right)\right] \\
& +e^{\lambda \tau} \sum_{j=1}^{n}\left[\left(\left|b_{\hat{k} j}^{R}\right| l_{j}^{R R}+\left|b_{\hat{k} j}^{I}\right| l_{j}^{I R}\right) V_{j}\left(t_{1}-\tau_{\hat{k} j}(t), \mathbf{z}\right)\right. \\
& \left.+\left(\left|b_{\hat{k} j}^{R}\right| l_{j}^{R I}+\left|b_{\hat{k} j}^{I}\right| l_{j}^{I I}\right) V_{j+n}\left(t_{1}-\tau_{\hat{k} j}\left(t_{1}\right), \mathbf{z}\right)\right] \\
& +\sum_{j=1}^{n}\left\{\int _ { - \infty } ^ { t _ { 1 } } \sigma _ { \hat { k } j } ( t _ { 1 } - s ) e ^ { \lambda ( t _ { 1 } - s ) } \left[\left(\left|q_{\hat{k} j}^{R}\right| l_{j}^{R R}+\left|q_{\hat{k} j}^{I}\right| l_{j}^{I R}\right) V_{j}(s, \mathbf{z})\right.\right. \\
& \left.\left.+\left(\left|q_{\hat{k} j}^{R}\right| l_{j}^{R I}+\left|q_{\hat{k} j}^{I}\right| l_{j}^{I I}\right) V_{j+n}(s, \mathbf{z})\right] \mathrm{d} s\right\} \\
& \leq\left\{\lambda-2 \frac{m \pi_{\hat{k}}}{\hat{\omega}^{2}}-2 d_{\hat{k}}+\sum_{j=1}^{n}\left[\left(l_{j}^{R R}+l_{j}^{R I}\right)\left(\left|a_{\hat{k} j}^{R}\right|+\left|b_{\hat{k} j}^{R}\right|+\left|q_{\hat{k} j}^{R}\right|\right)\right.\right. \\
& \left.\left.+\left(l_{j}^{I R}+l_{j}^{I I}\right)\left(\left|a_{\hat{k} j}^{I}\right|+\left|b_{\hat{k} j}^{I}\right|+\left|q_{\hat{k} j}^{I}\right|\right)\right]\right\} \beta_{\hat{k}} \chi_{0} \\
& +\sum_{j=1}^{n}\left[\left(\left|a_{\hat{k} j}^{R}\right| l_{j}^{R R}+\left|a_{\hat{k} j}^{I}\right| l_{j}^{I R}\right) \beta_{j} \chi_{0}+\left(\left|a_{\hat{k} j}^{R}\right| l_{\hat{k}}^{R I}+\left|a_{\hat{k} j}^{I}\right| l_{j}^{I I}\right) \gamma_{j} \chi_{0}\right] \\
& +e^{\lambda \tau} \sum_{j=1}^{n}\left[\left(\left|b_{\hat{k} j}^{R}\right| l_{j}^{R R}+\left|b_{\hat{k} j}^{I}\right| l_{j}^{I R}\right) \beta_{j} \chi_{0}\right. \\
& \left.+\left(\left|b_{\hat{k} j}^{R}\right| l_{j}^{R I}+\left|b_{\hat{k}_{j}}^{I}\right| l_{j}^{I I}\right) \gamma_{j} \chi_{0}\right] \\
& +\sum_{j=1}^{n}\left\{\mu_{\hat{k} j}(\lambda)\left[\left(\left|q_{\hat{k} j}^{R}\right| l_{j}^{R R}+\left|q_{\hat{k} j}^{I}\right| l_{j}^{I R}\right) \beta_{j} \chi_{0}+\left(\left|q_{\hat{k} j}^{R}\right| l_{j}^{R I}+\left|q_{\hat{k} j}^{I}\right| l_{j}^{I I}\right) \gamma_{j} \chi_{0}\right]\right\} \\
& =\left\{\lambda-2 \frac{m \pi_{\hat{k}}}{\hat{\omega}^{2}}-2 d_{\hat{k}}+\sum_{j=1}^{n}\left[\left(l_{j}^{R R}+l_{j}^{R I}\right)\left(\left|a_{\hat{k} j}^{R}\right|+\left|b_{\hat{k} j}^{R}\right|+\left|q_{\hat{k} j}^{R}\right|\right)\right.\right. \\
& \left.\left.+\left(l_{j}^{I R}+l_{j}^{I I}\right)\left(\left|a_{\hat{k} j}^{I}\right|+\left|b_{\hat{k} j}^{I}\right|+\left|q_{\hat{k} j}^{I}\right|\right)\right]\right\} \beta_{\hat{k}} \chi_{0} \\
& +\sum_{j=1}^{n}\left[\left(\left|a_{\hat{k} j}^{R}\right| l_{j}^{R R}+\left|a_{\hat{k} j}^{I}\right| l_{j}^{I R}\right)+\left(\left|b_{\hat{k} j}^{R}\right| l_{j}^{R R}+\left|b_{\hat{k} j}^{I}\right| l_{j}^{I R}\right) e^{\lambda \tau}\right. \\
& \left.+\left(\left|q_{\hat{k} j}^{R}\right| l_{j}^{R R}+\left|q_{\hat{k} j}^{I}\right| l_{j}^{I R}\right) \mu_{\hat{k} j}(\lambda)\right] \beta_{j} \chi_{0} \\
& +\sum_{j=1}^{n}\left[\left(\left|a_{\hat{k} j}^{R}\right| l_{\hat{k}}^{R I}+\left|a_{\hat{k} j}^{I}\right| l_{j}^{I I}\right)+\left(\left|b_{\hat{k} j}^{R}\right| l_{j}^{R I}+\left|b_{\hat{k} j}^{I}\right| l_{j}^{I I}\right) e^{\lambda \tau}\right. \\
& \left.+\left(\left|q_{\hat{k} j}^{R}\right| l_{j}^{R I}+\left|q_{\hat{k} j}^{I}\right| l_{j}^{I I}\right) \mu_{\hat{k} j}(\lambda)\right] \gamma_{j} \chi_{0}
\end{aligned}
$$

$<0$. 
As is apparent, there is a contradiction between (26) and $\mathrm{D}^{+}\left(V_{\hat{k}}\left(t_{1}, \mathbf{z}\right)\right) \geq 0$. Therefore, for all $t \geq 0$ and $\mathbf{z} \in \Omega$, we get $V_{k}(t, \mathbf{z})<\beta_{k} \chi_{0}$ and $V_{k+n}(t, \mathbf{z})<\gamma_{k} \chi_{0}$, which means that $V_{k^{\prime}}(t, \mathbf{z})<$ $\varsigma_{k^{\prime}} \chi_{0}$ and $k^{\prime} \in \widehat{\Theta}$.

(2) When $k^{\prime} \in \widehat{\Theta}-\Theta$, by calculating $\mathrm{D}^{+} V_{k^{\prime}}(t, \mathbf{z})$ along (19), and considering Assumptions 1-4 and Lemma 3, we have

$$
\begin{aligned}
& \mathrm{D}^{+} V_{k^{\prime}}(t, \mathbf{z}) \\
& =0.5 \lambda e^{\lambda t} \int_{\Omega}\left(u_{k}^{I}(t, \mathbf{z})\right)^{2} \mathrm{~d} \mathbf{z}+e^{\lambda t} \pi_{k} \sum_{h=1}^{m} \int_{\Omega} u_{k}^{I}(t, \mathbf{z}) \frac{\partial}{\partial z_{h}}\left[\frac{\partial u_{k}^{I}(t, \mathbf{z})}{\partial z_{h}}\right] \mathrm{d} \mathbf{z} \\
& +e^{\lambda t} \sum_{h=1}^{m} \int_{\Omega} u_{k}^{I}(t, \mathbf{z})\left\{-d_{k} u_{k}^{I}(t, \mathbf{z}) \sum_{j=1}^{n}\left[a_{k j}^{R} g_{j}^{I}\left(u_{j}^{R}(t, \mathbf{z}), u_{j}^{I}(t, \mathbf{z})\right)\right.\right. \\
& \left.+a_{k j}^{I} g_{j}^{R}\left(u_{j}^{R}(t, \mathbf{z}), u_{j}^{I}(t, \mathbf{z})\right)\right] \\
& +\sum_{j=1}^{n}\left[b_{k j}^{R} g_{j}^{I}\left(u_{j}^{R}\left(t-\tau_{k j}(t), \mathbf{z}\right), u_{j}^{I}\left(t-\tau_{k j}(t), \mathbf{z}\right)\right)\right. \\
& \left.+b_{k j}^{I} g_{j}^{R}\left(u_{j}^{R}\left(t-\tau_{k j}(t), \mathbf{z}\right), u_{j}^{I}\left(t-\tau_{k j}(t), \mathbf{z}\right)\right)\right] \\
& +\sum_{j=1}^{n}\left[q_{k j}^{R} \int_{-\infty}^{t} \sigma_{k j}(t-s) g_{j}^{I}\left(u_{j}^{R}(s, \mathbf{z}), u_{j}^{I}(s, \mathbf{z})\right) \mathrm{d} s\right. \\
& \left.\left.+q_{k j}^{I} \int_{-\infty}^{t} \sigma_{k j}(t-s) g_{j}^{R}\left(u_{j}^{R}(s, \mathbf{z}), u_{j}^{I}(s, \mathbf{z})\right) \mathrm{d} s\right]\right\} \mathrm{d} \mathbf{z} \\
& \leq\left\{\lambda-2 \frac{m \pi_{k}}{\hat{\omega}^{2}}-2 d_{k}+\sum_{j=1}^{n}\left[\left(l_{j}^{I R}+l_{j}^{I I}\right)\left(\left|a_{k j}^{R}\right|+\left|b_{k j}^{R}\right|+\left|q_{k j}^{R}\right|\right)\right.\right. \\
& \left.\left.+\left(l_{j}^{R R}+l_{j}^{R I}\right)\left(\left|a_{k j}^{I}\right|+\left|b_{k j}^{I}\right|+\left|q_{k j}^{I}\right|\right)\right]\right\} V_{k+n}(t, \mathbf{z}) \\
& +\sum_{j=1}^{n}\left[\left(\left|a_{k j}^{R}\right| l_{j}^{I R}+\left|a_{k j}^{I}\right| l_{j}^{R R}\right) V_{j}(t, \mathbf{z})+\left(\left|a_{k j}^{R}\right| l_{j}^{I I}+\left|a_{k j}^{I}\right| l_{j}^{R I}\right) V_{j+n}(t, \mathbf{z})\right] \\
& +e^{\lambda \tau} \sum_{j=1}^{n}\left[\left(\left|b_{k j}^{R}\right| l_{j}^{I R}+\left|b_{k j}^{I}\right| l_{j}^{R R}\right) V_{j}\left(t-\tau_{k j}(t), \mathbf{z}\right)\right. \\
& \left.+\left(\left|b_{k j}^{R}\right| l_{j}^{I I}+\left|b_{k j}^{I}\right| l_{j}^{R I}\right) V_{j+n}\left(t-\tau_{k j}(t), \mathbf{z}\right)\right] \\
& +\sum_{j=1}^{n}\left\{\int _ { - \infty } ^ { t } \sigma _ { k j } ( t - s ) e ^ { \lambda ( t - s ) } \left[\left(\left|q_{k j}^{R}\right| l_{j}^{I R}+\left|q_{k j}^{I}\right| l_{j}^{R R}\right) V_{j}(s, \mathbf{z})\right.\right. \\
& \left.\left.+\left(\left|q_{k j}^{R}\right| l_{j}^{I I}+\left|q_{k j}^{I}\right| l_{j}^{R I}\right) V_{j+n}(s, \mathbf{z})\right] \mathrm{d} s\right\}
\end{aligned}
$$

By a similar analysis to part (1) above, we demonstrate that, for all $t \geq 0, k \in \Theta, k^{\prime} \in \widehat{\Theta}-\Theta$, and the inequalities $V_{k}(t, \mathbf{z})<\beta_{k} \chi_{0}$ and $V_{k^{\prime}}(t, \mathbf{z})<\gamma_{k} \chi_{0}$ hold.

It can be summarized from (1) and (2) that $V_{k^{\prime}}(t)<\varsigma_{k^{\prime}} \chi_{0}, k^{\prime} \in \widehat{\Theta}$, which means that $0.5\left\|u_{k}^{R}(t, \mathbf{z})\right\|_{L_{2}}^{2} e^{\lambda t}<\beta_{k} \chi_{0}, 0.5\left\|u_{k}^{I}(t, \mathbf{z})\right\|_{L_{2}}^{2} e^{\lambda t}<\gamma_{k} \chi_{0}$, and $k \in \Theta$. 
Furthermore,

$$
\begin{aligned}
& \left\|u_{k}^{R}(t, \mathbf{z})\right\|_{L_{2}}^{2}<2 \theta \beta_{k} e^{-\lambda t}\|\boldsymbol{\varphi}(s, \mathbf{z})\|_{L_{2}}^{2} / \underline{v}, \\
& \left\|u_{k}^{I}(t, \mathbf{z})\right\|_{L_{2}}^{2}<2 \theta \gamma_{k} e^{-\lambda t}\|\boldsymbol{\varphi}(s, \mathbf{z})\|_{L_{2}}^{2} \underline{v}, \quad k \in \Theta .
\end{aligned}
$$

Let $\Gamma=\sqrt{2 \theta \bar{v} / \underline{v}}$, then we have

$$
\left\|u_{k}^{R}(t, \mathbf{z})\right\|_{L_{2}}<\Gamma\|\boldsymbol{\varphi}(s, \mathbf{z})\|_{L_{2}} e^{-0.5 \lambda t}, \quad\left\|u_{k}^{I}(t, \mathbf{z})\right\|_{L_{2}}<\Gamma\|\boldsymbol{\varphi}(s, \mathbf{z})\|_{L_{2}} e^{-0.5 \lambda t}, \quad k \in \Theta .
$$

According to Definition 1, the zero state of the RDCVNNs composed of (18) and (19) is globally exponentially stable and equals the global exponential stability of the equilibrium state of the RDCVNNs composed of (3) and (4). The proof is completed.

Remark 3 The scalar Lyapunov function method [8-11, 13, 18], the LMI method [6, 12, $14,16,17,19,21,23-25]$, and the fixed point theorem with inequality scaling skills [7] were adopted to judge the dynamical behaviors of various CVNNs. Due to the appearance of the free variables included in the criteria, the obtained criteria theoretically seem to exhibit low conservatism. As pointed out by [45], in actual applications, finding the perfect combination of supposed parameters and incoming free variables depends to a large degree on performing incessant attempts. The judging matrix $\tilde{\mathbf{T}}-\tilde{\mathbf{P}} \tilde{\mathbf{L}}$ in Theorem 2 is just composed of assumption conditions including diffusion coefficients, self-feedback coefficients, connection matrices, and some assumed parameters for the activation functions, which is simple to apply in practice.

Remark 4 If the reaction-diffusion terms are removed from model (1), the system considered in this paper is the same as that in model (1) in [5]. If only time-varying delays (let $\mathbf{Q}=\mathbf{0}$ ) or infinite distributed delays (let $\mathbf{B}=\mathbf{0}$ ) are considered in model (1), we can directly obtain the corresponding theorems to judge the existence, uniqueness, and global exponential stability of the equilibrium state of the system, respectively. In order to save space, we omitted this.

Based on the various applications of CVNNs, the complex-valued activation functions are usually classified into two categories. One of the two categories is made up of cases where the activation functions cannot be explicitly separated into their real and imaginary parts. The other category includes cases that are able to be expressed by separating their real and imaginary parts. Among the major results available presently, the first type is supposed to satisfy the Lipschitz condition (see Assumption 1 in [12, 15, 24]), and the related model is regarded as an integrity to analyze its dynamical behaviors based on the direct method, and the second type is supposed to satisfy Assumptions 4-5 in this paper. Next, we provide two additional common assumption conditions for activation functions, which have appeared in previous works.

Assumption 5 (see Assumption 3 in [12]) Let the activation function $\tilde{g}_{j}\left(\tilde{u}_{j}(t, \mathbf{z})\right)$ be divided into a real part and imaginary part as $\tilde{g}_{j}\left(\tilde{u}_{j}(t, \mathbf{z})\right)=\tilde{g}_{j}^{R}\left(\tilde{u}_{j}^{R}(t, \mathbf{z})\right)+i \tilde{g}_{j}^{I}\left(\tilde{u}_{j}^{I}(t, \mathbf{z})\right), j \in \Theta$. Assuming that there exist constants $l_{j}^{R-}, l_{j}^{R+}, l_{j}^{I-}$, and $l_{j}^{I+}$ such that, for any $\tilde{u}_{j}^{R}(t, \mathbf{z}) \neq \tilde{v}_{j}^{R}(t, \mathbf{z})$, we have

$$
l_{j}^{R-} \leq \frac{\tilde{g}_{j}^{R}\left(\tilde{u}_{j}^{R}(t, \mathbf{z})\right)-\tilde{g}_{j}^{R}\left(\tilde{v}_{j}^{R}(t, \mathbf{z})\right)}{\tilde{u}_{j}^{R}(t, \mathbf{z})-\tilde{v}_{j}^{R}(t, \mathbf{z})} \leq l_{j}^{R+}, \quad l_{j}^{I-} \leq \frac{\tilde{g}_{j}^{I}\left(\tilde{u}_{j}^{I}(t, \mathbf{z})\right)-\tilde{g}_{j}^{I}\left(\tilde{v}_{j}^{I}(t, \mathbf{z})\right)}{\tilde{u}_{j}^{I}(t, \mathbf{z})-\tilde{v}_{j}^{I}(t, \mathbf{z})} \leq l_{j}^{I+} .
$$


Let $l_{j}^{R}=\max \left\{\left|l_{j}^{R-}\right|,\left|l_{j}^{R-}\right|\right\}, l_{j}^{I}=\max \left\{\left|l_{j}^{I-}\right|,\left|l_{j}^{I-}\right|\right\}$, and $j \in \Theta$, and denote $\mathbf{L}^{R}=\operatorname{diag}\left(l_{j}^{R}\right)_{n \times n}$ and $\mathbf{L}^{I}=\operatorname{diag}\left(l_{j}^{I}\right)_{n \times n}$.

Theorem 3 Suppose that Assumptions 1-3 and Assumption 5 are satisfied. Then (1) has a unique equilibrium state for all $\mathbf{J} \in \mathbb{C}^{n}$ and $\mathbf{z} \in \Omega$ if the matrix $\tilde{\mathbf{T}}-\tilde{\mathbf{P}} \tilde{\mathbf{L}}$ is an M-matrix, where $\tilde{\mathbf{T}}=\operatorname{diag}\left(\tilde{T}_{k}\right)_{2 n \times 2 n}=\left[\begin{array}{ll}\mathbf{T} & 0 \\ 0 & \mathbf{T}\end{array}\right]$ and $\mathbf{T}=\operatorname{diag}\left(T_{k}\right)_{n \times n}$ with

$$
T_{k}=2 \frac{m \pi_{k}}{\hat{\omega}^{2}}+2 d_{k}-\sum_{j=1}^{n}\left[l_{j}^{R}\left(\left|a_{k j}^{R}\right|+\left|b_{k j}^{R}\right|+\left|q_{k j}^{R}\right|\right)+l_{j}^{I}\left(\left|a_{k j}^{I}\right|+\left|b_{k j}^{I}\right|+\left|q_{k j}^{I}\right|\right)\right], \quad k \in \Theta
$$

and

$$
\tilde{\mathbf{P}}=\left[\begin{array}{cc}
\left|\mathbf{A}^{R}\right|+\left|\mathbf{B}^{R}\right|+\left|\mathbf{Q}^{R}\right| & \left|\mathbf{A}^{I}\right|+\left|\mathbf{B}^{I}\right|+\left|\mathbf{Q}^{I}\right| \\
\left|\mathbf{A}^{I}\right|+\left|\mathbf{B}^{I}\right|+\left|\mathbf{Q}^{I}\right| & \left|\mathbf{A}^{R}\right|+\left|\mathbf{B}^{R}\right|+\left|\mathbf{Q}^{R}\right|
\end{array}\right], \quad \tilde{\mathbf{L}}=\left[\begin{array}{cc}
\mathbf{L}^{R} & \mathbf{0} \\
\mathbf{0} & \mathbf{L}^{I}
\end{array}\right] .
$$

The process of proving Theorem 3 is highly similar to that of Theorems $1-2$, so we have omitted it.

Remark 5 Although Assumption 5 removes the boundedness and continuity hypothesis for the partial derivatives of the activation functions with two variables (see Assumption 4 and related assumptions in $[5,14,26,28])$, the separation forms with respect to the activation functions that satisfy Assumption 5 are weak-coupled compared to the separation forms (2) described in this paper. That is to say, the two assumption conditions mentioned concerning activation functions have their own advantages and disadvantages, which means that it is necessary to discuss these two types of activation functions.

In (1), if $\tilde{u}_{k}(t, \mathbf{z}) \in \mathbb{R} \times \Omega(k \in \Theta)$ and the matrices $\mathbf{A}, \mathbf{B}$ and $\mathbf{Q}$ are defined in $\mathbb{R}^{n \times n}$, the RDCVNNs are changed into RDRVNNs. In this case, the corresponding criterion will be given to guarantee the existence, uniqueness, and global exponential stability of the equilibrium state of the system.

Corollary 1 Suppose that Assumptions 1-4 are satisfied. Then (1) has a unique equilibrium state for all $\mathbf{J} \in \mathbb{R}^{n}$ and $\mathbf{z} \in \Omega$ if the matrix $\mathbf{T}-(|\mathbf{A}|+|\mathbf{B}|+|\mathbf{Q}|) \mathbf{L}$ is an M-matrix, where $\mathbf{L}=\mathbf{L}^{R R}, \mathbf{T}=\operatorname{diag}\left(T_{k}\right)_{n \times n}$ with $T_{k}=2 \frac{m \pi_{k}}{\hat{\omega}^{2}}+2 d_{k}-\sum_{j=1}^{n} l_{j}\left[\left(\left|a_{k j}\right|+\left|b_{k j}\right|+\left|q_{k j}\right|\right)\right], k \in \Theta$.

\section{Numerical examples}

\subsection{Example 1}

Considering the RDCVNNs described as follows:

$$
\left\{\begin{aligned}
& \frac{\partial u_{k}(t, \mathbf{z})}{\partial t}= \pi_{k} \sum_{h=1}^{2} \frac{\partial}{\partial z_{h}}\left(\frac{\partial u_{k}(t, \mathbf{z})}{\partial z_{h}}\right)-d_{k} u_{k}(t, \mathbf{z})+\sum_{j=1}^{2}\left[a_{k j} g_{j}\left(u_{j}(t, \mathbf{z})\right)\right. \\
&\left.+b_{k j} g_{j}\left(u_{j}\left(t-\tau_{k j}(t)\right), \mathbf{z}\right)+q_{k j} \int_{-\infty}^{t} \sigma_{k j}(t-s) g_{j}\left(u_{j}(s, \mathbf{z})\right) \mathrm{d} s\right] \\
&\left.u_{k}(t, \mathbf{z})\right|_{\mathbf{z} \in \partial \Omega}=0
\end{aligned}\right.
$$

with the initial conditions $u_{1}^{R}(t, 0)=u_{2}^{R}(t, 0)=u_{1}^{I}(t, 0)=u_{2}^{I}(t, 0)=u_{1}^{R}(t, 8)=u_{2}^{R}(t, 8)=$ $u_{1}^{I}(t, 8)=u_{2}^{I}(t, 8)=0, t \geq 0$, and $u_{1}^{R}(0, \mathbf{z})=1.5 \sin \mathbf{z}, u_{1}^{I}(0, \mathbf{z})=\tanh \mathbf{z}, u_{2}^{R}(0, \mathbf{z})=\cos \mathbf{z}+1$, $u_{2}^{I}(0, \mathbf{z})=\cos 2 \mathbf{z}-0.5$, where $z_{h} \in(0,8), h=1,2$.

It is supposed that $\tau_{1 j}=0.35+0.2 \sin t, \tau_{2 j}=0.5+0.3 \cos t, \theta_{k j}(t-s)=\exp (-(t-s)), k, j=$ $1,2, t \geq 0, s \in(-\infty, 0]$. 
Let

$$
\begin{aligned}
& \mathbf{D}=\left[\begin{array}{cc}
8 & 0 \\
0 & 10
\end{array}\right], \quad \mathbf{A}=\left[\begin{array}{cc}
1-0.5 i & 1+i \\
-1-i & 2+0.5 i
\end{array}\right], \quad \mathbf{B}=\left[\begin{array}{cc}
1+i & 2-i \\
-1.5+i & 0.5-2 i
\end{array}\right], \\
& \mathbf{Q}=\left[\begin{array}{cc}
-2+i & -1-2 i \\
-1+i & 1-i
\end{array}\right], \quad \boldsymbol{\pi}=\left[\begin{array}{ll}
2 & 0 \\
0 & 1
\end{array}\right]
\end{aligned}
$$

and

$$
\begin{aligned}
& g_{1}\left(u_{1}\right)=\frac{1-\exp \left(-u_{1}^{R}\right)}{1+\exp \left(-u_{1}^{R}\right)}+i \frac{1}{1+\exp \left(-u_{1}^{I}(t, z)\right)}, \\
& g_{2}\left(u_{2}\right)=\frac{1-\exp \left(-u_{2}^{I}\right)}{1+\exp \left(-u_{2}^{I}\right)}+i \frac{1}{1+\exp \left(-u_{2}^{R}\right)} .
\end{aligned}
$$

By calculation, we have

$$
\mathbf{L}^{R R}=\left[\begin{array}{cc}
0.5 & 0 \\
0 & 0
\end{array}\right], \quad \mathbf{L}^{R I}=\left[\begin{array}{cc}
0 & 0 \\
0 & 0.5
\end{array}\right], \quad \mathbf{L}^{I R}=\left[\begin{array}{cc}
0 & 0 \\
0 & 0.25
\end{array}\right], \quad \mathbf{L}^{I I}=\left[\begin{array}{cc}
0.25 & 0 \\
0 & 0
\end{array}\right]
$$

Furthermore, it can be calculated that

$$
\begin{aligned}
\tilde{\mathbf{T}}-\tilde{\mathbf{P}} \tilde{\mathbf{L}}= & {\left[\begin{array}{cccc}
10.5 & 0 & 0 & 0 \\
0 & 14.9 & 0 & 0 \\
0 & 0 & 10.5 & 0 \\
0 & 0 & 0 & 14.9
\end{array}\right]-\left[\begin{array}{cccc}
4 & 4 & 2.5 & 4 \\
3.5 & 3.5 & 3 & 3.5 \\
2.5 & 4 & 4 & 4 \\
3 & 3.5 & 3.5 & 3.5
\end{array}\right] } \\
& \times\left[\begin{array}{cccc}
0.5 & 0 & 0 & 0 \\
0 & 0 & 0 & 0.5 \\
0 & 0 & 0.25 & 0 \\
0 & 0.25 & 0 & 0
\end{array}\right] \\
= & {\left[\begin{array}{cccc}
8.50 & -1.00 & -0.63 & -2.00 \\
-1.75 & 14.03 & -0.75 & -1.75 \\
-1.25 & -1 & 9.50 & -2.00 \\
-1.50 & -0.88 & -0.88 & 13.15
\end{array}\right] . }
\end{aligned}
$$

Because the eigenvalue vector of the matrix $\tilde{\mathbf{T}}-\tilde{\mathbf{P}} \tilde{\mathbf{L}}$ is $[6.69,10.00,13.58,14.90]$, it follows from Lemma 1 that the matrix $\tilde{\mathbf{T}}-\tilde{\mathbf{P}} \tilde{\mathbf{L}}$ is an M-matrix. Moreover, according to Theorems $1-2$, it can be concluded that the equilibrium state of (28) is existent, unique, and exponentially stable.

The numerical simulations of (28) are shown in Figs. $1-5$. Figure 1 and Fig. 3 show the state surfaces of the real parts in (28). Figure 2 and Fig. 4 show the state surfaces of the imaginary parts in (28). Figure 5 shows the state curves of $u_{1}^{R}(t, \mathbf{z}), u_{1}^{I}(t, \mathbf{z}), u_{2}^{R}(t, \mathbf{z})$, and $u_{2}^{I}(t, \mathbf{z})$ of (28) with different space values, which are referred to in the simulation results. From the simulation results shown in Figs. 1-5, it can be seen that the equilibrium state of (28) is existent, unique, and stable. 


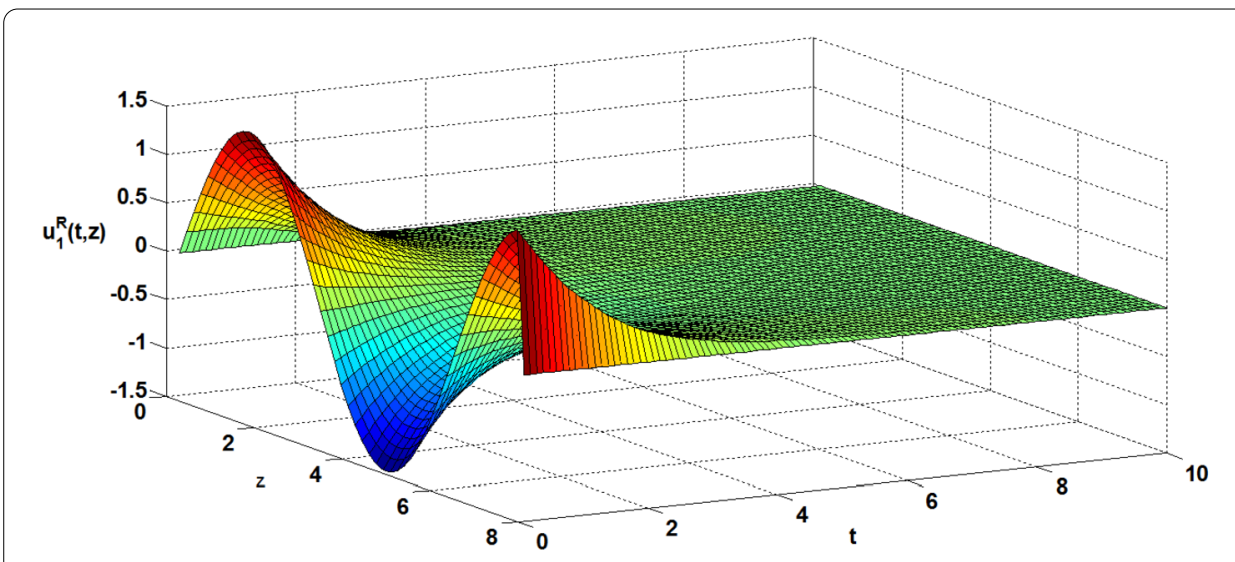

Figure 1 State curved surface of $u_{1}^{R}(t, \mathbf{z})$ in (28) with initial condition $u_{1}^{R}(0, \mathbf{z})=1.5 \sin \mathbf{z}$

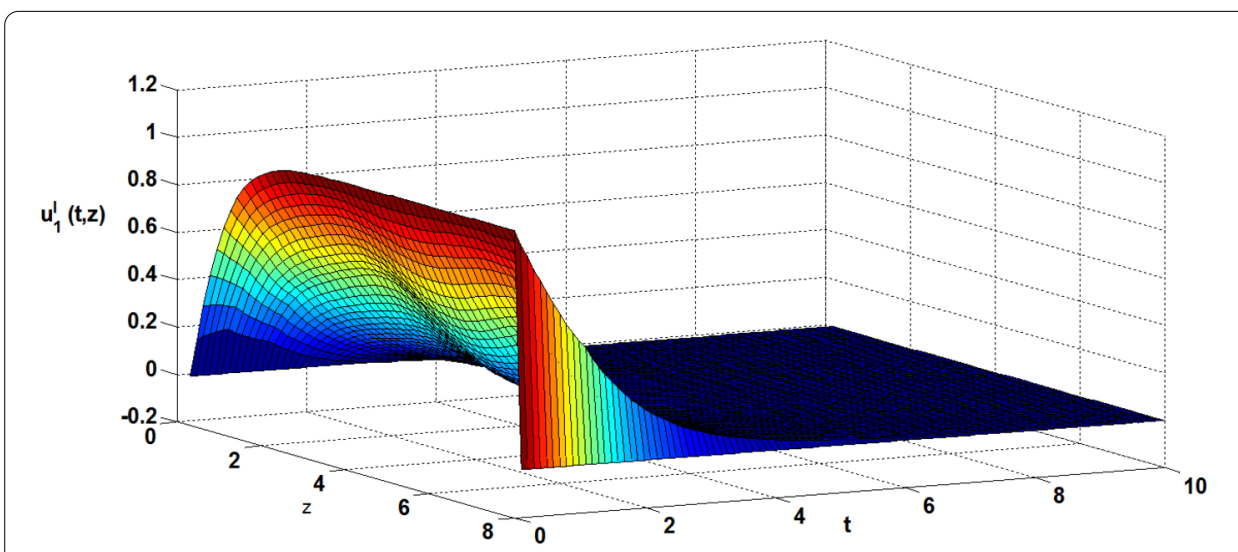

Figure 2 State curved surface of $u_{1}^{\prime}(t, \mathbf{z})$ in (28) with initial condition $u_{1}^{\prime}(0, \mathbf{z})=\tanh \mathbf{z}$

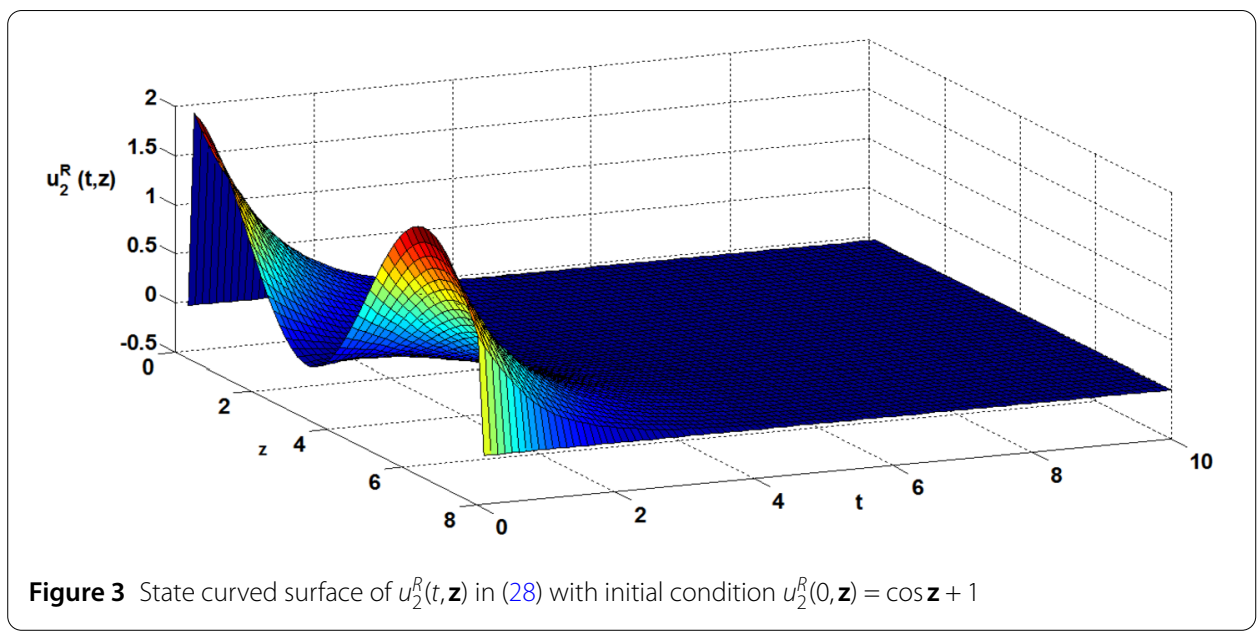

Remark 6 Some criteria for judging the stability of RDRVNNs have been established $[35,43,44]$. However, these criteria are independent of diffusion, which neglects the active effects of the reaction-diffusion terms. The proposed criteria in [30-34, 36, 37, 39, 40, 42] 

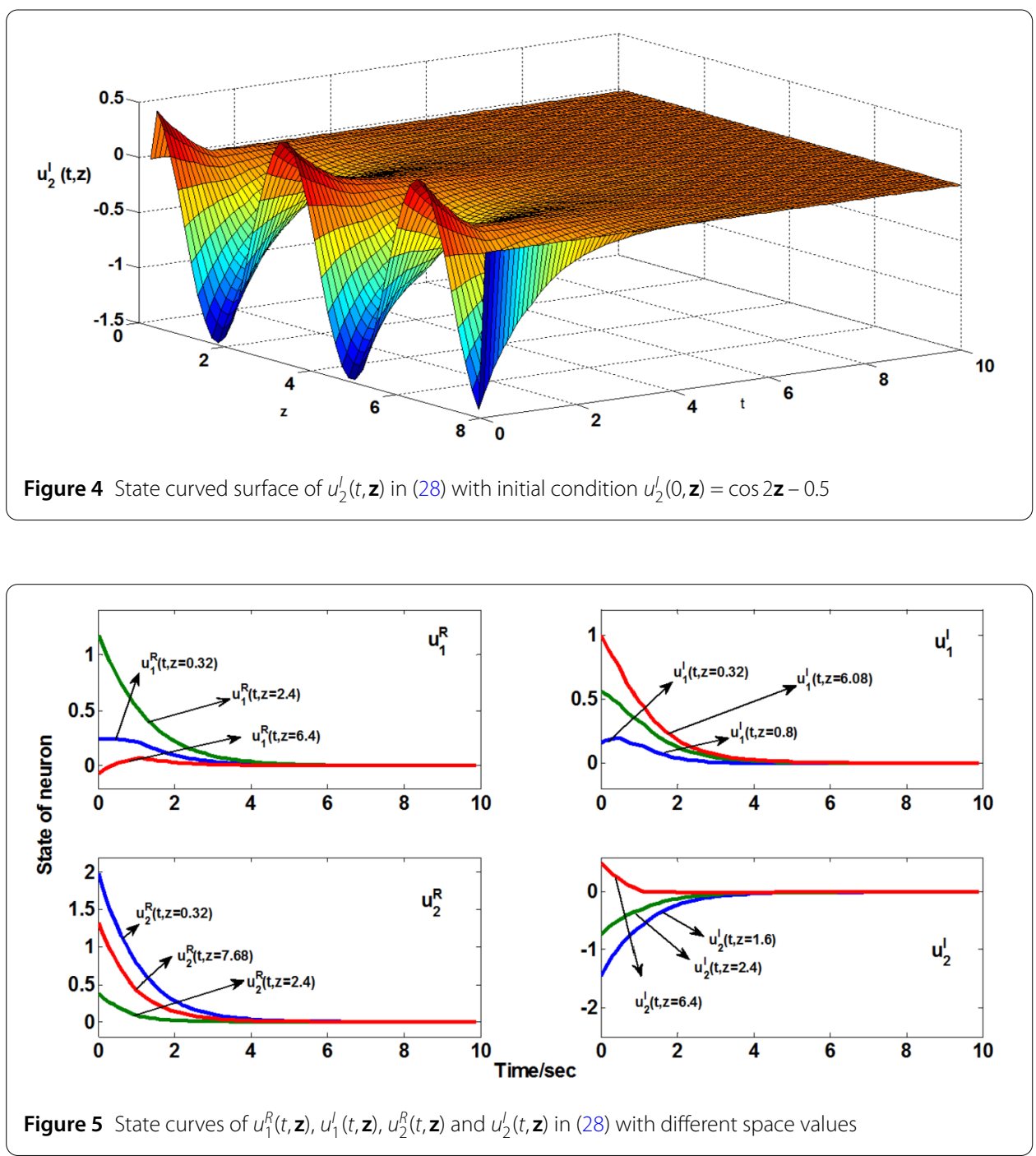

for RDRVNNs are diffusion-dependent. The results obtained [28, 29] for analyzing the dynamical behaviors of RDCVNNs are also diffusion-dependent. In order to further demonstrate the positive influence of reaction-diffusion terms on the stability, the following computational comparisons with simulation curves are provided to verify this opinion.

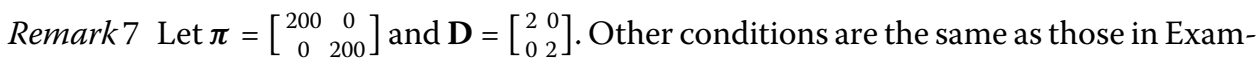
ple 4.1. If the positive effect of diffusion on stability is ignored, the matrix $\tilde{\mathbf{T}}$ is computed as

$$
\tilde{\mathbf{T}}-\tilde{\mathbf{P}} \tilde{\mathbf{L}}=\left[\begin{array}{cccc}
-1.63 & -1.00 & -0.63 & -2.00 \\
-1.75 & -1.13 & -0.75 & -1.75 \\
-1.25 & -1 & -1.63 & -2.00 \\
-1.50 & -0.88 & -0.88 & -1.13
\end{array}\right]
$$

Clearly, because the matrix $\tilde{\mathbf{T}}-\tilde{\mathbf{P}} \tilde{\mathbf{L}}$ is not an M-matrix, the exponential stability of the equilibrium state of (28) cannot be determined. However, if the positive effect of reaction- 


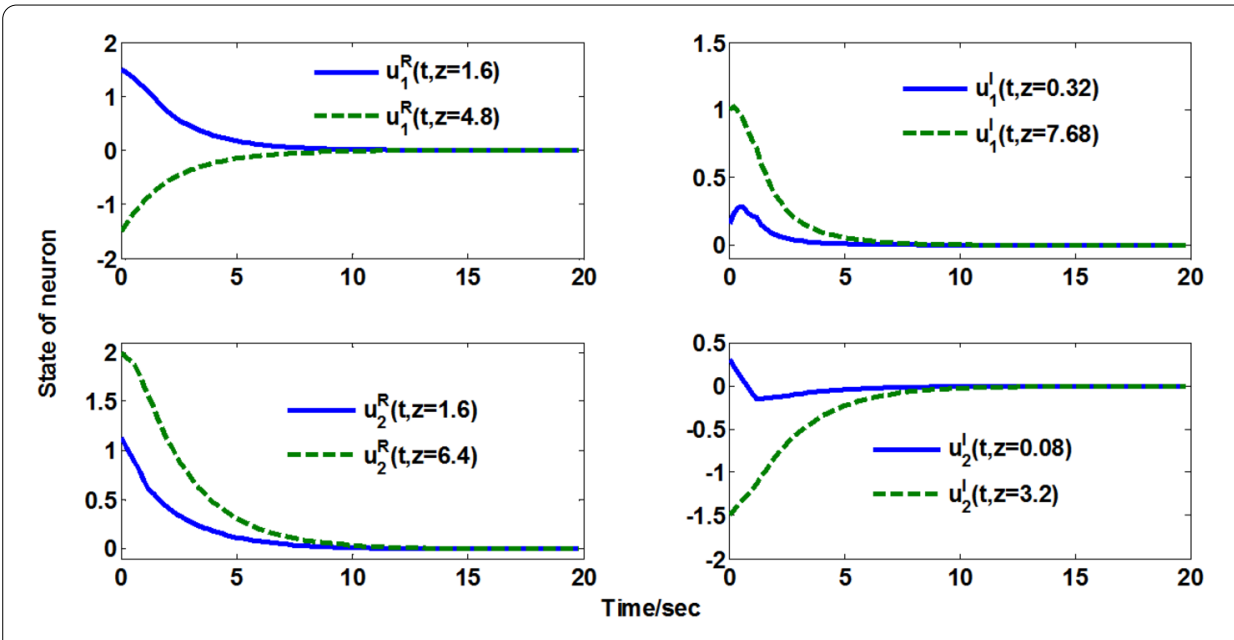

Figure 6 State curves $u_{1}^{R}(t, \mathbf{z}), u_{1}^{\prime}(t, \mathbf{z}), u_{2}^{R}(t, \mathbf{z})$ and $u_{2}^{\prime}(t, \mathbf{z})$ of (28) with different space values

diffusion terms on the stability is taken into account, we get

$$
\tilde{\mathbf{T}}-\tilde{\mathbf{P}} \tilde{\mathbf{L}}=\left[\begin{array}{cccc}
6.88 & -1.00 & -0.63 & -2.00 \\
-1.75 & 7.38 & -0.75 & -1.75 \\
-1.25 & -1 & 6.88 & -2.00 \\
-1.50 & -0.88 & -0.88 & 7.38
\end{array}\right]
$$

with the eigenvalue vector $[3.91,6.98,9.15,8.48]$. It is obvious that the equilibrium state of (28) is exponentially stable according to Theorem 2. The true state curves of (28) with different space values are shown in Fig. 6. From the simulated curves, we can claim that the equilibrium states of (28) exist and are unique and stable, although these are unable to be judged according to the conditions without including the positive effect of diffusion on the stability. That is to say, our results exhibit less conservatism as well as a wider scope for parameters.

\subsection{Example 2}

In order to make a comparison with Theorem 3.3 in [37] and Corollary 1 in [40], the following model is considered:

$$
\left\{\begin{aligned}
& \frac{\partial u_{k}(t, \mathbf{z})}{\partial t}= \sum_{h=1}^{3} \pi_{k h} \frac{\partial}{\partial z_{h}}\left(\frac{\partial u_{k}(t, \mathbf{z})}{\partial z_{h}}\right)-d_{k} u_{k}(t, \mathbf{z}) \\
&+\sum_{j=1}^{2}\left[a_{k j} g_{j}\left(u_{j}(t, \mathbf{z})\right)+b_{k j} g_{j}\left(u_{j}\left(t-\tau_{k j}(t)\right), \mathbf{z}\right)\right] \\
&\left.u_{k}(t, \mathbf{z})\right|_{\mathbf{z} \in \partial \Omega}=0 .
\end{aligned}\right.
$$

For the sake of calculation and to save space, we only provide some of the parameters and assumption conditions that are included in Theorem 3.3 [37], Corollary 1 [40], and Corollary 1 in this paper. It is supposed that $z_{h} \in[0,1], \pi_{1}=(0.1,0.2,0.3), \pi_{2}=(0.2,0.2,0.1)$, $g_{j}\left(u_{j}\right)=0.1 *\left(\left|u_{j}+1\right|-\left|u_{j}-1\right|\right), \tau_{1 j}(t)=1-0.5 \exp (-t), \tau_{2 j}(t)=1-0.3 \exp (-t), \mathbf{D}=\operatorname{diag}(1,1)$, and $\mathbf{A}=\mathbf{B}=\left[\begin{array}{ll}1 & 1 \\ 1 & 1\end{array}\right]$. 
(a) Let $p=2$. Choosing $\varepsilon=\lambda_{1}=\lambda_{2}=0.5$ and computing the conditions of Theorem 3.3 in [37] as follows:

$$
\left.\begin{array}{c}
2 \lambda_{1} \varepsilon-2 \lambda_{1} c_{1}-2 \lambda_{1} \sum_{k=1}^{3} \frac{a_{1 k}}{l_{k}^{2}}+\lambda_{1} \sum_{j=1}^{2}\left(\left|d_{1 j}\right|+\left|e_{1 j}\right|\right) F_{j} \\
\quad+\sum_{j=1}^{2} \lambda_{j}\left(\left|d_{j 1}\right|+\frac{\exp (2 \varepsilon \sigma)\left|e_{j 1}\right|}{1-\delta}\right) F_{1}=0.587>0 \\
2 \lambda_{2} \varepsilon-2 \lambda_{2} c_{2}-2 \lambda_{2} \sum_{k=1}^{3} \frac{a_{2 k}}{l_{k}^{2}}+\lambda_{2} \sum_{j=1}^{2}\left(\left|d_{2 j}\right|+\left|e_{2 j}\right|\right) F_{j} \\
\quad+\sum_{j=1}^{2} \lambda_{j}\left(\left|d_{j 2}\right|+\frac{\exp (2 \varepsilon \sigma)\left|e_{j 2}\right|}{1-\delta}\right) F_{2}=0.687>0
\end{array}\right\} .
$$

(b) Let $r_{i}\left(w_{i}(t, x)\right)=w_{i}(t, x)$ in (7) of [40], and let $p=2$ and $d_{i j}=0$ in (35) of [40]. Choosing $\varsigma=\tilde{m}_{1}=\tilde{m}_{2}=0.5$ and computing the conditions (36) extended from (35) in Corollary 1 of [40], the exponential stability of (29) can be determined as follows:

$$
\left.\begin{array}{l}
2 \tilde{m}_{1} \varsigma-2 \tilde{m}_{1} a_{1}-\sum_{k=1}^{3} \frac{2 \tilde{m}_{1} a_{1 k}}{l_{k}^{2}}+\sum_{j=1}^{2} \tilde{m}_{j}\left(2\left|b_{j 1}\right| F_{j}+\left|c_{1 j}\right| G_{j}\right) \\
\quad+\sum_{j=1}^{2} \tilde{m}_{j}\left(\frac{\exp (2 \varsigma \sigma)\left|c_{j 1}\right|}{1-\sigma}\right) G_{j}=0.159>0 \\
2 \tilde{m}_{2} \varsigma-2 \tilde{m}_{2} a_{1}-\sum_{k=1}^{3} \frac{2 \tilde{m}_{2} a_{2 k}}{l_{k}^{2}}+\sum_{j=1}^{2} \tilde{m}_{j}\left(2\left|b_{j 2}\right| F_{j}+\left|c_{2 j}\right| G_{j}\right) \\
\quad+\sum_{j=1}^{2} \tilde{m}_{j}\left(\frac{\exp (2 \varsigma \sigma)\left|c_{j 2}\right|}{1-\sigma}\right) G_{j}=0.259>0
\end{array}\right\} .
$$

(c) Computing the conditions of Corollary 1 provided in this paper, we get $\mathbf{T}-(|\mathbf{A}|+$ $|\mathbf{B}|) \mathbf{L}=\left[\begin{array}{cc}2.6 & -0.8 \\ -0.8 & 2.6\end{array}\right]$. It is obvious that the matrix $\mathbf{T}-(|\mathbf{A}|+|\mathbf{B}|) \mathbf{L}$ is an M-matrix.

Remark 8 From the above calculated results shown in (30) and (31), we find that the inequalities of Theorem 3.3 in [37] and Corollary 1 in [40] used to judge the exponential stability of the equilibrium state of (29) aren't satisfied, which means that Theorem 3.3 in [37] and Corollary 1 in [40] are unable to obtain the exponential stability of (29). However, the exponential stability of the states of (29) has been judged using Corollary 1 in this study. Based on Example 4.2, we claim that the result has a larger range for parameters than Theorem 3.3 in [37] and Corollary 1 in [40]. Moreover, the exponential stability conditions proposed in this paper are easier to verify than the conditions in $[37,40]$.

\section{Conclusions and future research}

A type of RDCVNNs with time-varying and infinite distributed delays was investigated in this study. By means of the homeomorphism theory and vector Lyapunov function method as well as M-matrix theory, some conditions were obtained to judge the existence, uniqueness, and global exponential stability of the systems. The established conditions include the positive influence of reaction-diffusion terms on the stability and reduce the conservatism of the existing ones. Several examples are provided to illustrate the feasibility and reduced conservatism of the established conditions compared to the present research. Besides of real-valued neural networks and CVNNs, an increasing number of studies are focusing on the quaternion-valued neural networks because of their advantages in aspect of image processing, unmanned aerial vehicle driving and recognition, human behavior recognition; see Refs. $[45,46]$ and the references therein. The method applied in this paper and the work done in [45] demonstrate a potential route for further research, which will concentrate on the robust stability for a type of delayed quaternion-valued NNs with reaction-diffusion term. 


\section{Acknowledgements}

The authors are grateful to the editor and the reviewers for their constructive comments and suggestions for the improvement of the paper.

\section{Funding}

This work has been supported in part by the Science \& Technology Major Project of Sichuan Province (Grant No. 2019ZDZX0002), in part by the Science \& Technology Department of Sichuan Province (Grant Nos. 21 QYCX0044 and 2019YFG0367), in part by the National Natural Science Foundation of China (Grant No. 51775448), in part by the Major Scientific \& Technological Innovation Project of Chengdu, Sichuan Province (Grant No. 2019-YF08-00003-GX), in part by the National Key R\&D Program of China (Grant No. 2018YFB1201603-11), in part by the Science \& Technology Department of Sichuan Province (Grant Nos. 2018GZ0110 and 2018HH0125), in part by the Key Research and Development Project of Sichuan Province (Grant No. 21ZDYF3520), in part by the Regional Cooperation and innovation project of Sichuan Province (Grant No. 2020YFQ0037), in part by the Scientific Research Foundation of the Education Department of Sichuan Province (Grant No. 18CZ0017), in part by the Key Scientific Research Fund Project of Xihua University (Grant No. Z202091), in part by the Open Research Subject of Key Laboratory of Fluid and Power Machinery, Xihua University, Ministry of Education (Grant No. szjj2019-015).

\section{Availability of data and materials}

All data generated or analyzed during this study are included in this paper.

\section{Competing interests}

The authors declare that they have no competing interests.

\section{Authors' contributions}

All authors contributed equally to the writing of this paper. All authors read and approved the final manuscript.

\section{Author details}

${ }^{1}$ Key Laboratory of Fluid and Power Machinery, Ministry of Education, Xihua University, Chengdu, 610039, China. ${ }^{2}$ Key Laboratory of Automobile Measurement and Control \& Safty, School of Automobile \& Transportation, Xihua University, Chengdu, 610039, China. ${ }^{3}$ School of Mechanical Engineering, Xihua University, Chengdu, 610039, China.

\section{Publisher's Note}

Springer Nature remains neutral with regard to jurisdictional claims in published maps and institutional affiliations.

\section{Received: 18 August 2020 Accepted: 14 December 2020 Published online: 20 January 2021}

\section{References}

1. Tanaka, G., Aihara, K.: Complex-valued multistate associative memory with nonlinear multilevel functions for gray-level image reconstruction. IEEE Trans. Neural Netw. 20, 1463-1473 (2009)

2. Wang, X.D., Che, M.L., Wei, Y.M.: Complex-valued neural networks for the Takagi vector of complex symmetric matrices. Neurocomputing 223, 77-85 (2017)

3. Xu, Q., Xu, X.H., Zhuang, S.X., Xiao, J.X., Song, C.H., Che, C.: New complex projective synchronization strategies for drive-response networks with fractional complex-variable dynamics. Appl. Math. Comput. 338, 552-566 (2018)

4. Hirose, A.: Complex-valued neural networks. In: Advances and Applications, vol. 18. Wiley, New York (2013)

5. Xu, X.H., Zhang, J.Y., Shi, J.Z: Exponential stability of complex-valued neural networks with mixed delays. Neurocomputing 128, 483-490 (2014)

6. Song, Q.K., Yu, Q.Q., Zhao, Z.J., Liu, Y.R., Alsaadi, F.: Boundedness and global robust stability analysis of delayed complex-valued neural networks with interval parameter uncertainties. Neural Netw. 103, 55-62 (2018)

7. You, X.X., Song, Q.K., Zhao, Z.J.: Existence and finite-time stability of discrete fractional-order complex-valued neural networks with time delays. Neural Netw. 123, 248-260 (2020)

8. Guo, R.N., Zhang, Z.Y., Liu, X.P., Lin, C.: Existence, uniqueness, and exponential stability analysis for complex-valued memristor-based BAM neural networks with time delays. Appl. Math. Comput. 311, 100-117 (2017)

9. Guo, R.N., Zhang, Z.Y., Liu, X.P., Lin, C., Wang, H.X., Chen, J.: Exponential input-to-state stability for complex-valued memristor-based BAM neural networks with multiple time-varying delays. Neurocomputing 275, 2041-2054 (2018)

10. Shi, Y.C., Cao, J.D., Chen, G.R.: Exponential stability of complex-valued memristor-based neural networks with time-varying delays. Appl. Math. Comput. 313, 222-234 (2017)

11. Wang, Z.Y., Liu, X.Z.: Exponential stability of impulsive complex-valued neural networks with time delay. Math. Comput. Simul. 156, 143-157 (2019)

12. Zhang, Z.Y., Liu, X.P., Chen, J., Guo, R.N., Zhou, S.W.: Further stability analysis for delayed complex-valued recurrent neural networks. Neurocomputing 251,81-89 (2017)

13. Wang, L.M., Song, Q.K., Liu, Y.R., Zhao, Z.J., Alsaadi, F.: Global asymptotic stability of impulsive fractional-order complex-valued neural networks with time delay. Neurocomputing 243, 49-59 (2017)

14. Zhang, Z.Q., Zheng, T.: Global asymptotic stability of periodic solutions for delayed complex-valued Cohen-Grossberg neural networks by combining coincidence degree theory with LMI method. Neurocomputing 289, 220-230 (2018)

15. Tang, Q., Jian, J.G.: Global exponential convergence for impulsive inertial complex-valued neural networks with time-varying delays. Math. Comput. Simul. 159, 39-56 (2019)

16. Jian, J., Wan, P.: Global exponential convergence of fuzzy complex-valued neural networks with time-varying delays and impulsive effects. Fuzzy Sets Syst. 338, 23-39 (2018)

17. Popa, C.: Global $\mu$-stability of neutral-type impulsive complex-valued BAM neural networks with leakage delay and unbounded time-varying delays. Neurocomputing 376, 73-94 (2020) 
18. Ali, M., Narayanan, G., Shekher, V., Alsaedi, A., Ahmad, B.: Global Mittag-Leffler stability analysis of impulsive fractional-order complex-valued BAM neural networks with time varying delays. Commun. Nonlinear Sci. Numer. Simul. 83, Article ID 105088 (2020)

19. Zhang, D.W., Jiang, H.J., Wang, J.L., Yu, Z.Y.: Global stability of complex-valued recurrent neural networks with both mixed time delays and impulsive effect. Neurocomputing 282, 157-166 (2018)

20. Xu, X.H., Zhang, J.Y., Shi, J.Z.: Dynamical behaviour analysis of delayed complex-valued neural networks with impulsive effect. Int. J. Syst. Sci. 48, 686-694 (2017)

21. Samidurai, R., Sriraman, R., Zhu, S.: Leakage delay-dependent stability analysis for complex-valued neural networks with discrete and distributed time-varying delays. Neurocomputing 338, 262-273 (2019)

22. Xu, D.S., Tan, M.C.: Multistability of delayed complex-valued competitive neural networks with discontinuous non-monotonic piecewise nonlinear activation functions. Commun. Nonlinear Sci. Numer. Simul. 62, 352-377 (2018)

23. Hu, B.X., Song, Q.K., Zhao, Z.J.: Robust state estimation for fractional-order complex-valued delayed neural networks with interval parameter uncertainties: LMI approach. Appl. Math. Comput. 373, Article ID 125033 (2020)

24. Cao, Y., Sriraman, R., Shyamsundarraj, N., Samidurai, R.: Robust stability of uncertain stochastic complex-valued neural networks with additive time-varying delays. Math. Comput. Simul. 171, 207-220 (2020)

25. Gunasekaran, N., Zhai, G.S.: Stability analysis for uncertain switched delayed complex-valued neural networks. Neurocomputing 367, 198-206 (2019)

26. Wang, P.F., Zou, W.Q., Su, H.: Stability of complex-valued impulsive stochastic functional differential equations on networks with Markovian switching. Appl. Math. Comput. 348, 338-354 (2019)

27. Ji, C.H., Qiao, Y.H., Miao, J., Duan, L.J.: Stability and Hopf bifurcation analysis of a complex-valued Wilson-Cowan neural network with time delay. Chaos Solitons Fractals 115, 45-61 (2018)

28. Dong, T., Bai, J.Q., Yang, L.: Bifurcation analysis of delayed complex-valued neural network with diffusions. Neural Process. Lett. 50, 1019-1033 (2019)

29. Huang, Y.L., Hou, J., Yang, E.F.: Passivity and synchronization of coupled reaction-diffusion complex-valued memristive neural networks. Appl. Math. Comput. 379, Article ID 125271 (2020)

30. Xu, X.H., Zhang, J.Y., Zhang, W.H.: Stochastic exponential robust stability of interval neural networks with reaction-diffusion terms and mixed delays. Commun. Nonlinear Sci. Numer. Simul. 17, 4780-4791 (2012)

31. Lu, X.M., Chen, W.H., Ruan, Z., Huang, T.W.: A new method for global stability analysis of delayed reaction-diffusion neural networks. Neurocomputing 317, 127-136 (2018)

32. Wang, Z.S., Zhang, H.G., Li, P.: An LMI approach to stability analysis of reaction-diffusion Cohen-Grossberg neural networks concerning Dirichlet boundary conditions and distributed delays. IEEE Trans. Syst. Man Cybern., Part B, Cybern. 40, 1596-1606 (2010)

33. Ma, Q., Feng, G., Xu, S.Y.: Delay-dependent stability criteria for reaction-diffusion neural networks with time-varying delays. IEEE Trans. Cybern. 43, 1913-1920 (2013)

34. Wu, K.N., Ren, M.Z., Liu, X.Z.: Exponential input-to-state stability of stochastic delay reaction-diffusion neural networks. Neurocomputing 412, 399-405 (2020)

35. Wang, Z.S., Zhang, H.G.: Global asymptotic stability of reaction-diffusion Cohen-Grossberg neural networks with continuously distributed delays. IEEE Trans. Neural Netw. 21, 39-49 (2010)

36. Yang, Z.C., Zhou, W.S., Huang, T.W.: Input-to-state stability of delayed reaction-diffusion neural networks with impulsive effects. Neurocomputing 333, 261-272 (2019)

37. Wang, J.L., Wu, H.N., Guo, L.: Passivity and stability analysis of reaction-diffusion neural networks with Dirichlet boundary conditions. IEEE Trans. Neural Netw. 22, 2105-2116 (2011)

38. Wang, J.L., Qiu, S.H., Chen, W.Z., Wu, H.N., Huang, T.W.: Recent advances on dynamical behaviors of coupled neural networks with and without reaction-diffusion terms. IEEE Trans. Neural Netw. Learn. Syst. (2020). https://doi.org/10.1109/TNNLS.2020.2964843

39. Suriguga, K.Y., Wang, C.H., Xia, H.W.: Robust mean square stability of delayed stochastic generalized uncertain impulsive reaction-diffusion neural networks. J. Franklin Inst. (2020). https://doi.org/10.1016/j.jfranklin.2020.04.011

40. Sheng, Y., Zhang, H., Zeng, Z.G.: Stability and robust stability of stochastic reaction-diffusion neural networks with infinite discrete and distributed delays. IEEE Trans. Syst. Man Cybern. Syst. 50, 1721-1732 (2020)

41. Wei, T.D., Lin, P., Wang, Y.F., Wang, L.S.: Stability of stochastic impulsive reaction-diffusion neural networks with S-type distributed delays and its application to image encryption. Neural Netw. 116, 35-45 (2019)

42. Wang, T.Y., Zhu, Q.X.: Stability analysis of stochastic BAM neural networks with reaction-diffusion, multi-proportional and distributed delays. Phys. A, Stat. Mech. Appl. 533, Article ID ID121935 (2019)

43. Balasubramaniam, P., Vidhya, C.: Global asymptotic stability of stochastic BAM neural networks with distributed delays and reaction-diffusion terms. Comput. Appl. Math. 234, 3458-3466 (2010)

44. Balasubramaniam, P., Vidhya, C.: Exponential stability of stochastic reaction-diffusion uncertain fuzzy neural networks with mixed delays and Markov jumping parameters. Expert Syst. Appl. 39, 3109-3115 (2012)

45. Xu, X.H., Xu, Q., Yang, J.B., Xue, H.B., Xu, Y.H.: Further research on exponential stability for quaternion-valued neural networks with mixed delays. Neurocomputing 400, 186-205 (2020)

46. Ji, X.R., Lu, J.Q., Lou, J.G., Qiu, J.L., Shi, K.B.: A unified criterion for global exponential stability of quaternion-valued neural networks with hybrid impulses. Int. J. Robust Nonlinear Control 30, 8098-8116 (2020) 